\title{
Noisy Perceptual Expectations: Multiple Object Tracking Benefits When Objects Obey Features of Realistic Physics
}

\author{
Jonas Sin-Heng Lau, Timothy F. Brady \\ Department of Psychology \\ University of California, San Diego
}

Please address correspondence to:

Timothy F. Brady

Department of Psychology

University of California, San Diego

9500 Gilman Drive \#0109

La Jolla, CA, 92093

Email: timbrady@ucsd.edu 


\begin{abstract}
When objects move, their motion is governed by the laws of physics. We investigated whether multiple objects that move while correctly obeying aspects of Newtonian physics are easier to track than those that do not accurately obey the laws of physics. Participants were asked to track multiple objects that either did or did not take on the correct angles and/or speeds after collisions with each other. We found an advantage for tracking when objects obeyed realistic physics, such that people were more accurate when objects reflected from each other at proper angles and when objects varied in speed after collisions (as opposed to always maintaining the same speed). This advantage was independent of a variety of low-level factors that would be expected to affect object tracking, such as object spacing. However, we also found that performance was not affected when objects' speed changed randomly after each collision (so long as it varied), nor when the reflection angles were jittered moderately after collisions. We conclude that perceptual noise seriously limits many aspects of object trajectory estimation, but nevertheless people are sensitive to at least a subset of the Newtonian laws of physics under demanding attentional tracking conditions.
\end{abstract}

Keywords: Intuitive Physics, Multiple Object Tracking (MOT), Visual Attention

\title{
Significance Statement
}

Asking people to track multiple objects that bounce randomly around a screen ('multiple object tracking', MOT) has been extensively utilized in the study of visual attention in the past 30 years. Visual attention researchers have used this method to understand how object positions are updated in our mind as they move, and other cognitive scientists have used it as a tool to understand general mental processing and executive control, such as how visual attention interacts with visual working memory, or factors that affect people's driving ability while engaging in a cell-phone conversation. In the current study, we show that contrary to people's own intuitions, they are better able to track objects when the objects they track obey Newtonian physics than when the objects move at a steady, constant speed, even though the constant speed motion is in many ways simpler and more predictable. This suggests that people automatically use their intuitive sense of object physics while tracking, and that to accurately gauge people's visual attention capacity, object physics should be taken into account. 
PHYSICS IN TRACKING

\section{Introduction}

People have expectations about the physics of how objects move even when they do not have formal scientific training in physics. Playing with marbles and toy cars in early childhood, we know that a collision of two moving objects will lead to a change in their trajectories, which can be decomposed into independent speed and direction components. Understanding the physics of such motion is important for day-to-day functioning, as we need to have a basic understanding of gravity and friction to play ball games, prevent rolling objects from falling off the desk, or avoid bumping into other objects in the environment. Here we ask whether our day-to-day experience with object motion also plays a role in attentional tracking of objects as they move, a common and important task (e.g., tracking other cars while driving; or tracking soccer players as they run on the field). In particular, we ask to what extent expectations about the motion of objects in collisions affect people's ability to track objects under extremely attentionally demanding conditions.

The classical Multiple Object Tracking paradigm (MOT; Pylyshyn \& Storm, 1988) offers a means to estimate people's expectations about the physics of object motion under cognitively demanding conditions. In a typical MOT experiment, participants are asked to track a number of moving objects among other visually identical distractors. Most MOT experiments take place in a simplified situation with no gravity and no friction, because adding such elements would in general make the task too simple (as the objects would simply stop). In an attempt to closely control for low-level factors, such as speed and spacing, in most MOT experiments, objects also do not obey even straightforward laws of the physics of how objects collide (e.g., elastic collisions). In particular, most studies have objects move at a constant speed, or with a haphazard pattern (e.g., Cooke, ter Horst, van Beers, \& Medendorp, 2017; Vul, Frank, Alvarez, \& Tenenbaum, 2009). Thus, when objects collide, they nevertheless continue to move at the same (fixed) speed. In some instantiations, objects pass through each other without colliding (e.g., Franconeri, Pylyshyn, \& Scholl, 2012; Horowitz \& Cohen, 2010; Vul et al. 2009). While important for 
PHYSICS IN TRACKING

matching low-level factors across displays, these behaviors do not capture some of the features that are representative of object interactions in a naturalistic environment.

In the current study, we modified the classical MOT paradigm to ask about the role of object collision physics in tracking. We kept the absence of gravity and friction typically used in these tasks, as this is necessary to make these tasks cognitively demanding (with gravity and/or friction, all the balls would simply stay in one place). However, we ask whether people have a higher capacity for tracking objects when they follow more realistic physics, particularly a correct model of object collisions. Might people's tracking ability differ with a more realistic physics of object collisions -- and what factors might affect this? Below, we briefly review the existing literature that bears on this question.

\section{People show some sensitivity to real world physics}

A set of theories that examine object motion among different populations conclude that children are sensitive to physical laws of object motion early in their development (e.g., Carey \& Xu, 2001; Scholl \& Leslie, 1999). This is true even in other animals (Flombaum et al. 2004), and literature on decision making involving intuitive physics has shown that people can make accurate predictions about the trajectories of moving objects based on mental simulations (e.g., Hamrick, Battaglia, \& Tenenbaum, 2011; Keane and Pylyshyn, 2006; Smith, Battaglia \& Vul, 2018), suggesting a rich intuitive physics engine for predicting object motion. Based on this, we might expect a benefit for tracking objects that obey physical laws, since their trajectories fit with what people have learned about the physical world and what is expected according to their intuitive physics of object collisions. In the field of cognitive psychology, it has been found that information congruent to that in the mental model is processed with greater efficiency (e.g., Eriksen, \& Eriksen, 1974; Stroop, 1935). This is especially the case if participants have the cognitive resources to simulate the outcome in such a cognitively demanding situation as multiple object tracking. 
PHYSICS IN TRACKING

\section{Extrapolation of motion in multiple object tracking}

An important factor for whether the physics of object collisions will be relevant to multiple object tracking performance is the extent to which people are aware of the patterns of object motion at all while doing the task. For example, when people are doing cognitively demanding multiple object tracking, do they extrapolate the motion trajectories of objects? The dominant view in the field of visual attention suggests that the physics of object motion, insofar as it does not impact low-level factors like spacing, should have no effect on MOT (e.g., Scimeca \& Franconeri, 2015). In particular, studies have found that in demanding MOT tasks, people have very limited attention capacity to extrapolate motion trajectories (Howe \& Holcombe, 2012; Keane \& Pylyshyn 2006), implying they may simply follow the objects with their attention without regard to the exact pattern of their motion or expectations about what the objects will do in the future.

In a computational simulation study, Zhong, Ma, Wilson, Liu, and Flombaum (2014) employed a Kalman filter to simulate object tracking performance under different conditions. In a critical experiment, the "50/50 Prediction Model", which is capable of extrapolating motion trajectories, was pitched against the "Spatial Working Memory Model", which does not extrapolate. Performance of the two models were nearly identical, and both were similar to performance of human observers. The results suggest that even if human observers extrapolate object trajectories during tracking, the relatively large perceptual noise makes the process largely ineffective. It is worth noting, though, some of the features of the object motion in their stimuli made extrapolation difficult. When objects came close together, they changed directions to avoid collision, and the direction was randomly determined without making reference to the law of physics. Thus, it remains somewhat unclear what such a model would predict with more straightforward motion physics. 
PHYSICS IN TRACKING

This absence of evidence on extrapolation is consistent with reports from the intuitive physics literature that people generate only 3 to 7 simulations when they have to make slow, reasoned judgements about object movements (Battaglia, Hamrick \& Tenenbaum, 2013). Such a limited capacity for object physics simulations would imply that people are unlikely to generate simulations fast enough in an MOT task, where multiple objects constantly interact with each other and change directions many times per second. Hence, the physics of motion may have little effect on tracking performance (e.g., Franconeri, Pylyshyn, \& Scholl, 2012; Horowitz, Birnkrant, Fencsik, Tran, \& Wolfe, 2006; Keane \& Pylyshyn, 2006).

Yet some evidence has found that participants do sometimes extrapolate trajectories, at least to some limited extent. For example , lordanescu, Grabowecky and Suzuki (2009) had participants track 3 of 10 objects, while all objects moving slowly according to the principle of elastic collisions. At the end of the trial, all objects disappeared. Participants had to click on the locations of the targets on the blank screen. The authors found that observers did not click on the exact locations where the objects disappeared, but on positions that would be predicted if the objects continued to move for a short period of time. This showed that observers were sensitive to the direction, and to a lesser extent, speed of the object motion. They also found that motion direction and speed are monitored independently (but see Howard, Masom, \& Holcombe, 2011). Similarly, Fencsik, Klieger, and Horowitz (2007) showed that when participants track 1 to 4 objects moving at constant speed, and the objects sometimes disappeared, participants performed best when given a preview of the motion before the objects disappeared, again indicating utilization of motion trajectories at least for small set sizes. Thus, at least in some circumstances, participants do appear to make use of some aspects of motion trajectories to predict where objects will be next. This opens the possibility that people are able to make use of realistic object collision dynamics to improve performance, even if they do not always extrapolate with more 
PHYSICS IN TRACKING

challenging motion trajectories (Zhong et al. 2014) or under all circumstances (Howe \& Holcombe, 2012).

\section{Variability in speed}

In addition to extrapolation, another aspect of multiple object tracking relevant to realistic physical situations is variability in the speed of the objects -- as opposed to all objects moving at constant speed, which is typical in multiple object tracking studies, realistic collisions cause objects to move at different speeds. Some studies in the literature suggest that this could impact tracking, but it is unclear from the literature whether this variability is beneficial. On the other hand, objects moving at a constant speed are, in an important low-level sense, much more predictable, as there is less object-specific information that needs to be tracked, and so there could be a benefit for this simpler pattern of motion common in the MOT literature over that of realistic physics. On the other hand, such variability could also improve tracking by providing another feature to track -- e.g., if all the targets are moving quickly at a given moment, people could use this global feature to segment the display.

Existing evidence is consistent with both possibilities. Meyerhoff, Papenmeier, Jahn, and Huff (2016) designed a study to test whether people benefit from tracking objects moving at a constant speed, compared to objects that moved at less predictable speeds. When objects moved at variable speeds, they followed a sine wave pattern for acceleration and deceleration. Therefore, the average speed of an object is matched between the two conditions. Tracking performance was found to be lower when object speed was variable, compared to when it was constant. By manipulating the number of objects moving at a variable speed, they found a linear decrease in performance as the number of variable speed objects went up.

Another line of research that looked at speed consistency concerns whether local or global features affect tracking performance. In St. Clair, Huff and Seiffert (2010), participants tracked multiple 
PHYSICS IN TRACKING

textured objects against a textured background. Objects were first defined at the beginning, and were motion-defined during the trial. When the objects moved, the pattern of the objects were either static, shifting to the same direction of the motion, orthogonal to the motion, or opposite to the motion. Tracking performance was maintained when the pattern shifted in the same direction as the object motion, but decreased in the orthogonal or opposite conditions. This shows that consistency between the local pattern on an object interacts with object motion aids tracking, and tracking performance suffers when there is a discrepancy between the two (see also Meyerhoff, Papenmeier, \& Huff, 2013; and Meyerhoff, Papenmeier, \& Huff, 2017 for a tutorial review).

\section{The current studies}

To disentangle these conflicting predictions and test the role of realistic object collision physics in MOT, Experiment 1 included two critical conditions. In one condition, participants tracked a subset of 8 moving objects that followed a constant speed model. Objects maintained the same speed after colliding with each other or bouncing off the enclosed boundary. In the other condition, the task was identical, except that the moving objects followed a realistic physics model, where objects sped up or slowed down and changed directions as appropriate from the collisions they underwent. If people are more accurate when objects move according to a realistic physics model, we can infer that people are sensitive to at least some features of Newtonian physics. That would provide support for the idea of a 'physics engine' in the mind that is used to interacting with the world (Hamrick, Battaglia, \& Tenenbaum, 2011), and provide evidence against purely passive attentional tracking without extrapolation of any kind to future object trajectories (e.g., Howe \& Holcombe, 2012). In Experiment 2, we tested conditions that dissociated the contributions of varying speed and reflection angles in the realistic physics model. The experiment suggests that people are not sensitive to the exact object speed after collision, but may be affected by post-collision reflection angles that are highly incorrect according to Newtonian physics. Interestingly, when asked to gauge their ability to track objects under a constant speed and realistic 
PHYSICS IN TRACKING

physics conditions, participants incorrectly believed they were more accurate at tracking when objects followed a predictable and constant speed, even though in fact they were better at tracking moving objects when the motion was governed by a realistic physics model. Thus, the intuitions participants have about their MOT performance do not accurately predict their performance. In the last two experiments, we showed that people are tolerant to small deviations of post-collision trajectories, as would be expected even from an accurate internal intuitive physics model if there was a perceptual noise in people's estimates of the objects (e.g., Hamrick et al. 2011).

Altogether, then, we suggest that while people have quite noisy perceptual estimates of objects in the MOT task, they do use the estimates of each object's pre-collision speed and pre-collision angle to make noisy predictions about the objects' trajectories after collisions, and thus benefit when the objects correctly follow realistic physical collision rules. The benefit cannot be attributed to low-level visual properties such as object-to-object distance or number of collisions, providing evidence that people have a sense of the physics of object collisions that goes above and beyond these low-level factors.

\section{Experiment 1A:}

\section{Realistic physics vs. constant object speed across-participants}

\section{Method}

\section{Design}

Experiment $1 \mathrm{~A}$ aimed to test how elements of realistic physics affected multiple object tracking abilities. Two groups of participants were shown 8 objects in each trial. In one of the groups, the objects always moved according to a constant speed model, and in the other group, object motion followed a realistic (Newtonian) physics model (see below for details). Participants were asked to track either 2 or 4 of the 8 moving objects as targets in a trial. Previous studies suggest that people's object tracking performance 
PHYSICS IN TRACKING

declines substantially if asked to track more than 4 or 5 objects (Intriligator \& Cavanagh, 2001; Pylyshyn \& Storm, 1988; Yantis, 1992). Motion physics was manipulated between-subjects, and the number of targets were manipulated within-subjects. The experiment was programmed with custom JAVA code.

\section{Participants}

Based on pilot data, we expected a large effect size (Cohen's $d \approx 0.8$ ). To achieve a power of $80 \%$ at such an effect size, at least 26 participants per condition, or a total of 52 participants, were required. We decided to recruit at least 30 participants per group to satisfy the normality assumption required by the statistical tests.

Sixty-two participants ( 44 female) were eventually recruited from the University of California, San Diego's Psychology Subject Pool, as we ran all subjects that were scheduled until we had a minimum of 60 . The participants had a mean age of 20.8 years at the time of their participation. All participants gave informed consent according to a protocol approved by the UCSD IRB prior to their participation and they participated for course credits.

\section{Apparatus and Stimuli}

Participants were individually placed in sound attenuated rooms with normal indoor lighting. Stimuli were shown on a Dell E173FPc 17-in LCD monitor with 4:3 aspect ratio. At a viewing distance of $60 \mathrm{~cm}$, the monitor's visible area was $31.2^{\circ}$ wide and $25.4^{\circ}$ tall in visual angle. The resolution of the screen was set to $1024 \times 768$. The visible region of the screen was cropped, such that only the central rectangular area of the screen was utilized. The rectangular window measured 824 pixels in width and 568 pixels in height, which translated to $25.32^{\circ}$ and $17.60^{\circ}$ in visual angle.

Eight circles were utilized in each trial. Each circle had a diameter of 50 pixels $\left(1.56^{\circ}\right.$ in visual angle). 
PHYSICS IN TRACKING

\section{Procedure}

Figure 1a illustrates a typical trial. Each trial began with a 1000ms blank screen, with the central rectangle appearing on the screen. Then the eight objects appeared on the screen for $3000 \mathrm{~ms}$. The initial positions of the objects were randomly selected, bounded by the rectangle, with the constraint that the objects could not overlap with each other. On any given trial, two or four of the 8 objects were designated as the targets. These targets appeared in blue and they blinked on and off slowly for $3000 \mathrm{~ms}$. The other objects remained gray throughout the interval. After this $3 \mathrm{~s}$ interval, all of the objects then turned gray and started moving at a speed of $5 \mathrm{px} /$ frame. The direction of the initiation motion was randomly determined for each object.

We computed trajectories assuming all objects had equal mass and the objects were moving on a frictionless surface. The equal mass assumption was consistent with the idea that all the objects appeared visually identical. The motion models that we chose did not factor in gravity, instead treating the display as an apparent birds-eye view on the objects moving on a horizontal surface - in line with previous multiple object tracking studies. Initial speed for each object was set to $5 \mathrm{px} /$ frame; each frame was $10 \mathrm{~ms}$, so the initial speed was $15.52 \%$ second (comparable to Alvarez \& Cavanagh, 2005). The objects reflected from each other when they were in contact with each other. They also bounced off the boundary of the central rectangle. As we assumed an elastic collision physics model, and objects moved in linear motion until they collided with one another or the boundary, the speed and direction of objects did not change from this linear trajectory except during collisions. This contrasts with a set of experiments in the literature which allowed less predictable changes in speed and direction in motion (e.g., Vul, Alvarez, \& Tenenbaum, 2009; Zhong, Ma, Wilson, Liu, \& Flombaum, 2014).

For participants in the constant speed condition, the motion vectors (speeds and directions) of the two colliding objects simply exchanged. That is, when two objects collided, they exchanged their motion directions (Figure 1b). Objects retained the same speed after they collided with each other or 
PHYSICS IN TRACKING

with the boundaries of the rectangle. For participants in the realistic physics condition, the change in object motion was calculated according to the law of momentum conservation, and thus objects took on speeds that deviated from their initial speeds (See Figure 1c for detail. Sample videos, pseudo-code of the motion, and full code of the experiment are included in the Appendix). The animation lasted 7500ms (Sample videos of Constant Speed condition: https://osf.io/ztmfa; Realistic Physics condition: https://osf.io/xewbn).

When the animation stopped, the computer cursor appeared. Participants were instructed to click on the objects to indicate the ones that they had been tracking. If they changed their mind during the selection process, they had the opportunity to make a correction. Participants were not allowed to submit any partial responses. Once the number of chosen objects matched with the number of targets in the trial, the cursor disappeared from the screen and feedback was shown for $3000 \mathrm{~ms}$.

Participants went through 10 practice trials before the experiment. During the experiment, each of the 5 blocks had 30 trials, with a 30-second break enforced between the blocks. Trials that required participants to track either 2 or 4 targets were mixed within a block, and the order was randomly determined. At the end of the experiment, participants were thanked and debriefed. The whole experiment lasted around 50 minutes. 


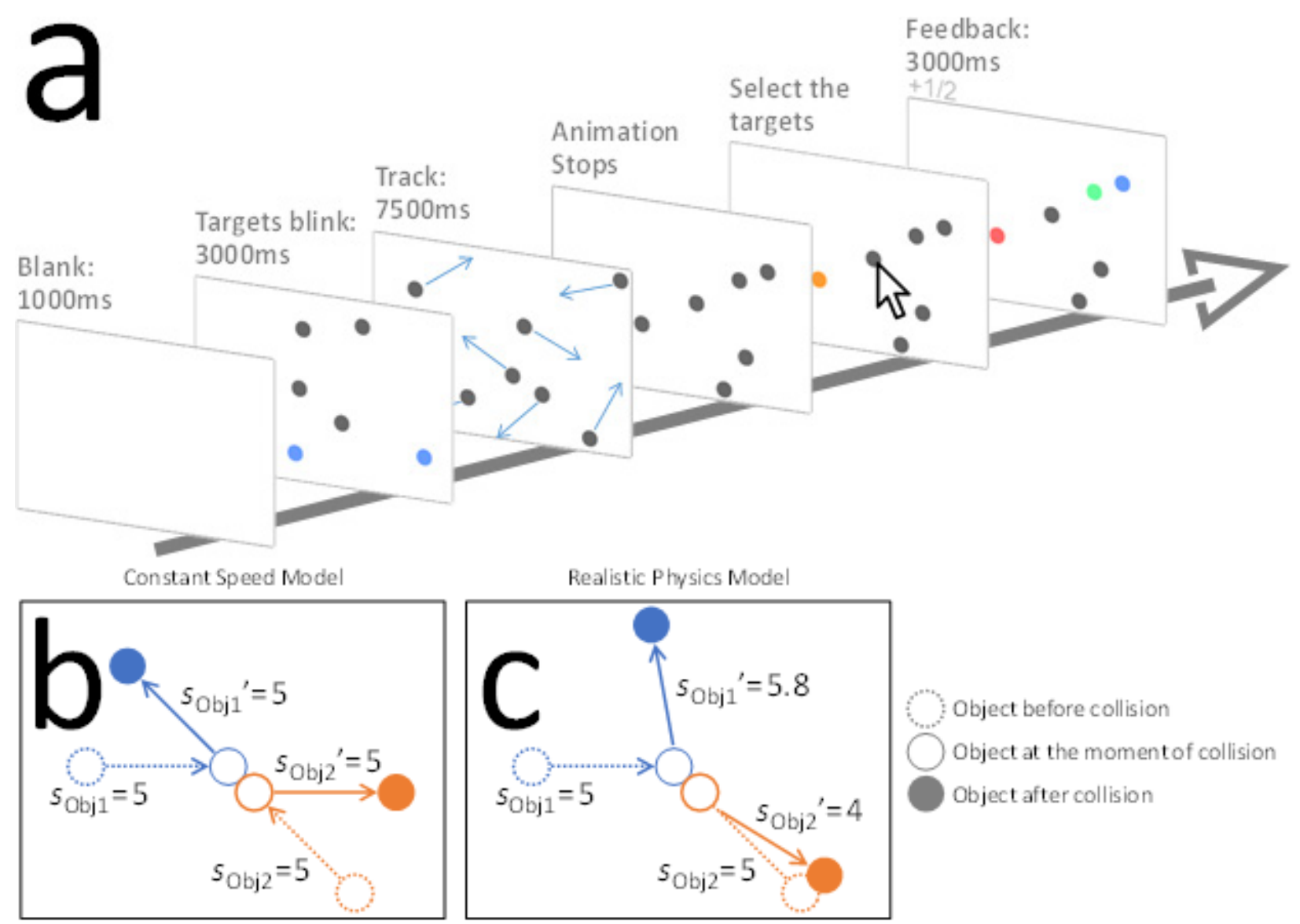

Figure 1. (a) General procedure in each trial. First, a subset of objects (2 or 4 of 8 ) is cued. Then these objects move for $7500 \mathrm{~ms}$ according to either a constant motion speed model or a realistic physics model. Finally, the animation stops and participants respond by selecting the objects they have been tracking. They are then given feedback. Targets correctly identified are shown in green, while distractors incorrectly selected as targets (false alarms) are shown in red. Targets missed are shown in blue. A score at the top-left hand corner indicates the number of targets a participant correctly identified in the trial. (b) A cartoon figure illustrating pre- and post-collision motion in the Constant Speed Condition. Object speeds are always the same before and after collision. The motion vectors (speed and direction) of two colliding objects simply exchange. Colors in the figure illustrate object identities, but in the task all objects were identical in appearance. (c) Motion in the Realistic Physics Condition, with pre-collision object locations, speeds, and motion directions identical to those in (b). Post-collision velocity (speed and direction) of an object is governed by Newtonian Physics. In both Constant Speed and Realistic Physics conditions, objects moved along a linear path without changing their speeds or direction, until they were in contact with another object or the boundary. Compared to (b), post-collision speeds of the objects can vary. The angles of reflection are also different from that in (b), even when objects in the two scenarios start with the same parameters. See Appendix for the pseudo-code of the computation, and sample videos of the two conditions.

\section{Results}

Data was submitted to a $2 \times 2$ ANOVA, with number of targets as the within-subjects factor and type of motion physics as the between-subjects factor. As expected, participants attained a higher accuracy 
PHYSICS IN TRACKING

when they were tracking 2 objects $(M=88.9 \%, S D=7.4 \%)$, compared to tracking 4 objects $(M=83.4 \%$, $\mathrm{SD}=7.9 \%), F(1,60)=45.12, p<0.001$, Cohen's $d_{z}=0.84$

Importantly, participants shown motion with realistic physics performed better $(\mathrm{M}=88.9 \%, \mathrm{SD}=$ 7.1\%) than those who tracked objects with a constant speed model $(\mathrm{M}=83.3 \%, \mathrm{SD}=5.6 \%), F(1,60)=$ 12.1, $p<0.001$, Cohen's $d=0.88$. There was no significant interaction between number of targets and the motion physics, $F(1,60)=1.86, p=0.18$. This suggests that participants did not use strongly divergent strategies when tracking 2 or 4 objects.

Thus, in Experiment 1A, we found initial evidence for a reliable benefit for realistic object collision physics over constant speed object motion. This benefit was quite substantial, with participants in the realistic physics condition performing as well tracking 4 objects as the participants in the constant speed condition did with just 2 objects. This is surprising given both previous evidence showing people do not generally extrapolate trajectories and given the more complex nature of the realistic physics condition: objects are all moving at distinct speeds, and there is more variability in the pattern of speeds and bounces. 


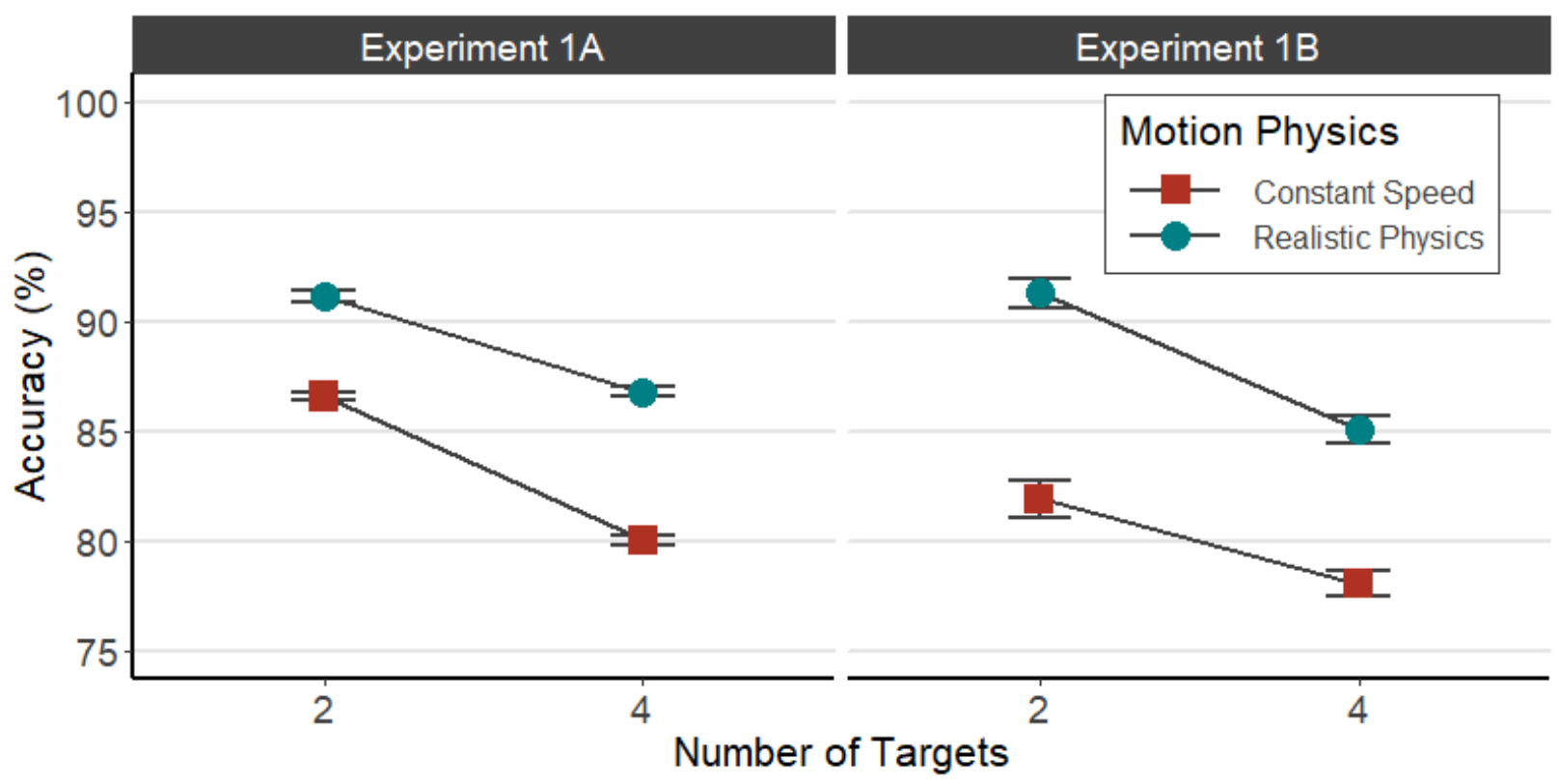

Figure 2. Results of Experiments $1 A$, the between-subjects manipulation of motion physics (left panel), and $1 B$, the within-subject manipulation of motion physics (right panel). In both conditions of the experiment, participants were more accurate when the objects followed a realistic physics model, compared to a constant speed model. Error bars denote standard errors around the means.

\section{Experiment 1B:}

\section{Realistic physics vs. constant object speed within-participants}

\section{Method}

\section{Design}

The goal of Experiment 1B was to replicate the results of Experiment 1a with a full within-subjects design, in preparation for a more complicated within-subjects design in Experiment 2. Thus, in Experiment 1B, both motion physics and the number of tracked objects were manipulated withinsubjects.

\section{Participants}

Participants were recruited from the same subject pool described above. With an estimated effect size of 0.8 and a desired power at $80 \%$, our power analysis indicated that around 15 participants were 
PHYSICS IN TRACKING

needed for a within-subjects design. Nevertheless, we aimed to recruit 30 participants. Thirty-one participants ( 23 female) were recruited. They had a mean age of 20.5 years at the time of participation. All participants took part in the study for course credits.

\section{Apparatus, Materials and Procedure}

The experimental set up was identical to that of Experiment $1 \mathrm{~A}$. After practice, participants completed 3 blocks of trials, each with 40 trials. In half of all trials, the objects moved according to the constant speed model; in the other half, their movements followed a realistic physics model. Participants were asked to track 2 targets in half of all trials, and 4 targets in the rest of the trials. The two factors were manipulated orthogonally. The order of these trials were randomized and mixed within a block.

\section{Results}

Accuracy was submitted to a $2 \times 2$ repeated-measures ANOVA, with number of targets and motion physics as the factors. The findings mirrored those of Experiment 1A. Performance was higher when participants tracked 2 targets $(M=86.8 \%, S D=7.4 \%)$, compared with tracking 4 targets $(M=81.7 \%$, SD $=8.8 \%), F(1,30)=43.3, p<0.001$, Cohen's $d_{z}=1.22$. Importantly, as in Experiment $1 \mathrm{~A}$, performance was higher when the object motion followed a realistic physics model $(M=88.2 \%, S D=7.5 \%)$, rather than a constant speed model $(\mathrm{M}=80.0 \%, \mathrm{SD}=8.9 \%), F(1,30)=94.2, p<0.001$, Cohen's $d_{z}=1.74$. The interaction between number of targets and object physics did not reach statistical significance, $F(1,30)=$ $2.48, p=0.13$. Thus, once again we found an advantage for tracking objects that moved according to realistic physics, even in this cognitively demanding context, and it was again similar when tracking 2 objects vs. 4 objects, suggesting it was not strongly modulated by cognitive load.

\section{Experiment 2: Dissociating speed from reflection angle}

Experiments $1 \mathrm{~A}$ and $1 \mathrm{~B}$ unequivocally showed that objects following realistic physics are easier to track than those moving according a constant speed model that is prevalent in the multiple object tracking 
PHYSICS IN TRACKING

literature. However, there are several possible reasons for this. One obvious candidate is speed. After the first object collisions, objects in the realistic physics condition move at new speeds. Some may have moved very quickly - becoming very difficult to track - while others moved slowly, becoming easier to track. In addition, since speed could vary reliably between different items until the next collision, it might have been utilized as an extra feature to set targets and distractors apart in the realistic physics condition, which could lead to an improvement in tracking (Papenmeier, Meyerhoff, Brockhoff, Jahn, \& Huff, 2017; St. Clair et al, 2010).

Thus, before examining low-level factors that may impact tracking (e.g., the extent to which the two different motion physics conditions differ in simple low-level factors, like, how nearby distractors tend to get to targets or how many collisions there are), we first sought to test whether varying speed alone is sufficient to improve tracking. In particular, we created two new "varied speed" conditions. The two conditions were identical to the constant speed and realistic physics conditions, respectively, except that a new speed was randomly assigned to an object after it collided with another object. The speed was drawn from a Gaussian distribution with a mean of $5 \mathrm{px} /$ frame and a standard deviation of 2 px/frame and was chosen independently after each collision. Thus objects had varied speeds but not predictable speeds, and the speeds could not be predicted based on any expectations from the collisions. In Experiment 1, when two objects in the constant speed model collided with another one, they simply exchanged their motion vectors. As a consequence, the objects did not retain proper reflection angles that would be expected from Newtonian physics. The "varied speed" model modified from the constant speed model inherited this property, hence we termed it "varied speed, incorrect angle" condition. On the other hand, when objects collided with each other in the realistic physics condition, the new velocities of the two objects were determined by an elastic collision model, assuming conservation of energy. The objects thus followed the angles that would be expected from Newtonian physics. This varied speed model modified from the realistic physics model inherited this property, 
PHYSICS IN TRACKING

hence we termed it "varied speed, correct angle" condition (Sample videos for "Varied Speed, Correct Angle" condition: https://osf.io/t8jpb; "Varied Speed, Incorrect Angle": https://osf.io/tdq37).

Thus, Experiment 2 aimed to both replicate the findings of Experiments $1 \mathrm{~A}$ and $1 \mathrm{~B}^{\prime}$ s constant speed and realistic physics conditions, as well as test the influence of speed on tracking performance with these two new conditions where speed was artificially randomized after each collision.

Our hypotheses were straightforward. If the improved performance in the realistic physics condition was completely attributed to the varied speed of the objects, all three conditions with different speeds among moving objects should support equally high performance (realistic physics, and the two randomized speed conditions). If proper reflection angles are needed in addition to varied speed, only the "varied speed, correct angle" condition and the realistic physics condition should support a high tracking performance. If all aspects of physics must be correctly preserved, only the realistic physics condition should support high performance.

\section{Method}

\section{Design}

The experiment included four different motion models: constant speed, "varied speed, incorrect angle", "varied speed, correct angle", and realistic physics. Participants tracked either two or four targets in each trial. Every participant encountered all eight possible conditions, and trials of different conditions were mixed within each block. As the constant speed and realistic physics conditions were identical to those in Experiment 1B, the main comparisons of interest were between the two new conditions and the two in the previous experiments.

\section{Participants}

Participants were recruited from the same subject pool described above. Forty participants (30 female) were recruited. They had a mean age of 20.6 years at the time of participation. All participants took part in the study for partial course credits. 
PHYSICS IN TRACKING

\section{Apparatus, Materials and Procedure}

The procedure was identical to that of Experiment 1B. Participants also completed an exit survey before the experiment concluded. The survey asked them to rate the difficulty of the tracking tasks.

\section{Results}

\section{Tracking Accuracy}

Accuracy were submitted to a $2 \times 4$ repeated-measures ANOVA, with number of tracked targets and type of motion physics as the factors. The results are summarized in Figure 3.

In general, participants performed better when they tracked 2 objects ( $M=84 \%, S D=17 \%)$ compared to 4 objects $(M=79 \%, S D=11 \%), F(1,39)=23.32, p<0.001$, Cohen's $d_{z}=0.94$. The pattern was consistent across the different motion physics conditions, suggested by a non-significant interaction between the two factors, $F(3,117)=1.26, p=0.29$. Since the interaction between the two factors was negligible, the analysis that follows will collapse the number of targets and focuses on the differences between the conditions with different motion physics.

The critical comparisons in this experiment were how the two varied speed conditions compared with the two conditions of Experiment 1 (constant speed and realistic physics). Results in the six pairwise comparisons were adjusted using the Bonferroni method to control for the overall Type I error. The mean accuracies for the constant speed, "varied speed, incorrect angle", "varied speed, correct angle", and realistic physics conditions were 79\% (SD = 13\%), 79\% (13\%), 85\% (12\%), and 85\% (11\%) respectively. Replicating Experiment $1 \mathrm{~A}$ and $1 \mathrm{~B}$, tracking accuracy was higher for the realistic physics condition, compared to the constant speed condition, $\mathrm{t}(39)=5.40, p_{a d j}<0.001$, Cohen's $d_{\mathrm{z}}=0.85$.

Varying speed alone, but with the incorrect reflection angles from the constant speed condition, did not improve tracking accuracy, as participants performed similarly in the constant speed and the "varied speed, incorrect angle" conditions, $\mathrm{t}(39)=0.11, p_{\text {adj }}=0.91$, Cohen's $d_{z}=0.02$, Bayes Factor $=5.83$ in favor of the null hypothesis. However, when the motion had varying speed and correct reflection 
angles, as in the "varied speed, correct angle" condition, performance was improved from the constant speed condition, $\mathrm{t}(39)=5.08, p_{a d j}<0.001$, Cohen's $d_{\mathrm{z}}=0.80$. Comparing the two varied speed conditions, imposing correct reflection angles enhanced tracking ability, $\mathrm{t}(39)=5.93, p_{\text {adj }}<0.001$, Cohen's $d_{z}=0.94$.

In fact, the accuracy of the realistic physics condition was indistinguishable to that of the "varied speed, correct angles" condition, $\mathrm{t}(39)=0.47, p_{a d j}=1$, Cohen's $d_{z}=0.07$, Bayes Factor $=5.28$ in favor of the null hypothesis. Similar to that in the "varied speed, correct angles" condition, accuracy in the realistic physics condition was higher than that in the "varied speed, incorrect angles", $t(39)=7.57$, $p_{a d j}<0.001$, Cohen's $d_{z}=1.20$

The results indicate that varying speed alone from the constant speed condition is not sufficient to improve tracking ability. People seem to be sensitive to the reflection angles after object collision. Once these two factors are in place, having the realistic speed after object collision does not further improve tracking accuracy. This pattern was replicated in an additional experiment, reported briefly in the Appendix.

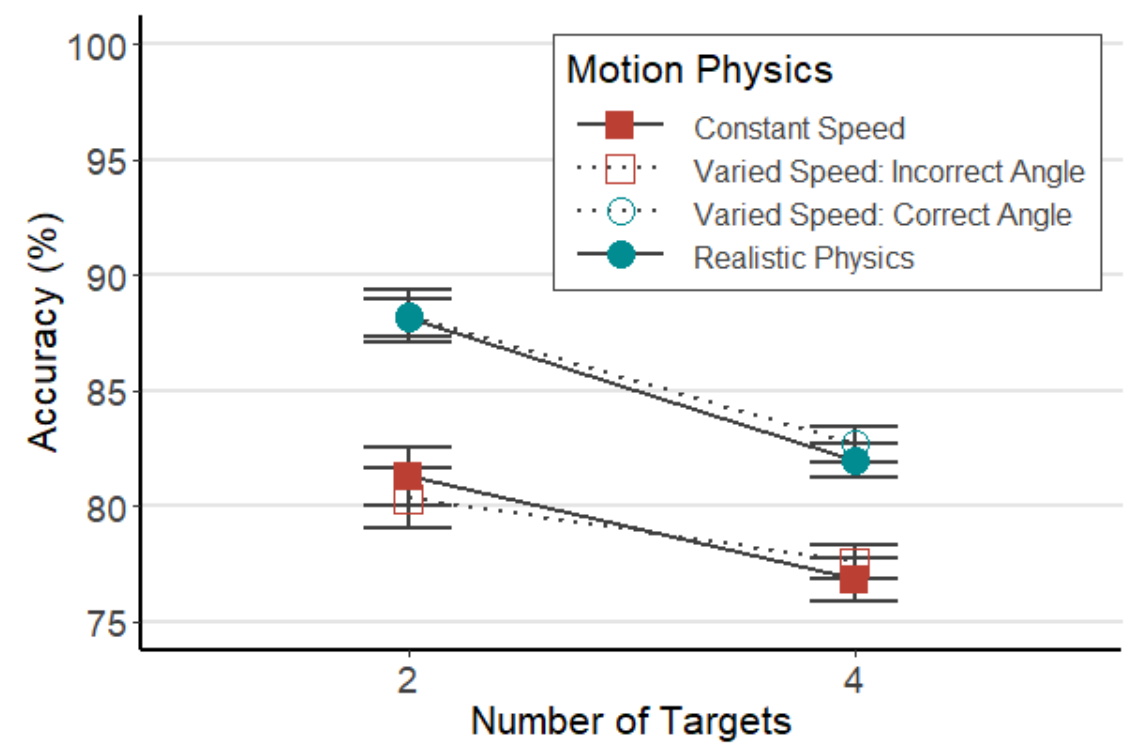

Figure 3. Results of Experiment 2. The "varied speed, incorrect angle" condition was modified from the constant speed model, where each item got a random speed (chosen from a normal distribution) after each collision. The "varied speed, correct angle" condition was modified from the realistic physics model in the same manner (that is, speeds varied but not according to proper physics). Accuracies of the two 
PHYSICS IN TRACKING

varied speed conditions were indistinguishable from their corresponding original models, suggesting that varying speed alone was not sufficient to improve performance.

\section{Perceived difficulties of the conditions}

At the end of the experiment, we asked participants whether tracking objects with constant or variable speed was easier. We did not ask more specifically which condition was harder because the varied speed and the realistic physics conditions could be hard to distinguish. When a participant answered "variable", he or she might refer to one of the varied speed conditions, or the realistic physics condition. With 40 participants, 30 (75\%) reported that tracking objects with constant speed was easier, 5 (12.5\%) reported that variable speed conditions were easier, and the rest (12.5\%) did not give a clear answer, $\chi^{2}(2)=31.25, p=0.001$. Thus, participants' reports contradict the data: In fact, the constant speed condition had a lower accuracy compared to the average of the three varied speed conditions, yet, perhaps because the objects appeared to move in more predictable patterns, participants believed this condition was easier. This provides evidence that the realistic physics advantages are unlikely to be a result of explicit, verbalizable problem solving (e.g., Smith, Battaglia, \& Vul, 2018).

\section{Simulation 1: Examining low-level factors}

Experiments 1 and 2 show that people perform better in MOT tasks when the objects they are tracking obey some aspects of realistic physics: in particular, they are better when objects vary in speed regardless of whether the objects' speeds are accurate according to physics - but only if those objects also follow correct reflection angles. However, by changing the motion physics in Experiments 1 and 2, it is possible that some low-level motion properties have also been altered. In particular, there might be differences in the spatial (Franconeri, et al, 2008; Franconeri, Jonathan, \& Scimeca, 2010), temporal (Holcombe \& Chen, 2013) or collision profiles of motion in these different conditions. If this was the case, it might be argued that benefits seen in the realistic physics and "varied speed, correct angle" conditions arise not from the intuitive sensitivity to correct real-world regularities in motion, but from 
PHYSICS IN TRACKING

simple low-level motion properties. For instance, if objects tend to keep a closer distance from one another in the constant speed condition, compared to the realistic physics condition, this alone could explain performance. Thus, to examine such low-level motion properties, we computed cumulative distributions of various low-level factors to study the objects and their motion in the four conditions. We focus on the differences between the two varied speed conditions, since the algorithms used to generate the two conditions differ only minimally, but the difference in performance was substantial. Overall, we find no evidence that low-level factors account for the pattern of data we observe. In particular, the two varied speed conditions result in nearly identical spatial, temporal and collision profiles, despite their large difference in behavioral performance.

Note that in multiple object tracking, participants performance is probably most impacted by the moments where objects collide with each other and thus can potentially be confused. Thus, the performance difference between the conditions would have the most to do with the speed and angle of the objects in the moments right before and after collisions. However, in our experiments, the moving objects always followed a linear path and maintained their moving speed and direction until they had another collision. Thus, the speeds of the objects would not change between immediately after a collision, and, say, 2 seconds after the collision, or immediately before the next collision. Thus, in these simulations, we look at object motion parameters across the entire time course of the tracking period, rather than focusing solely on the moments of collisions.

\section{Method}

We simulated 400 trials of each condition in Experiment 2 and recorded low-level properties of the displays and the objects' motion at different time points. To examine the spatial profiles of the conditions, pairwise distances of all the 8 objects were recorded when motion began, and when it was 2 seconds, 4 seconds, and 6 seconds into the motion. At each time point, $C_{2}^{8}=28$ data points were recorded, each representing the distance between two objects. The numbers were then tallied across 
PHYSICS IN TRACKING

the 400 trials for each condition. For each time point, we present the cumulative frequency curve for each condition. Curves that are closer to the left denote shorter object-to-object distance in general, which should increase object-tracking difficulty in that condition.

To examine the temporal profile of the conditions, a similar procedure was applied. Instead of object-to-object distance, we recorded each object's speed at a given time point. Eight data points were collected at each time point in each trial. A cumulative distribution curve that is closer to the right denotes overall higher speed in the condition, which should increase object tracking difficulty.

The collision distribution function was generated in a similar manner. The frequency that each object collided with one another within a trial's time was recorded. Eight data points were generated in each trial. The numbers from all 400 trials from the same condition formed one cumulative frequency curve. A condition with a cumulative distribution function closer to the right denotes more collisions, suggesting objects in this condition might be harder to track.

\section{Results}

Spatial, temporal and collision profiles of the four conditions are summarized in Figures 4 and 5 . As can be seen in Figure 4 (left panel), the spatial profiles of the four conditions are indistinguishable. This means objects are unlikely to be closer or farther away from each other in any one condition compared to the other conditions.

The profile of the objects' speed is plotted in Figure 4 (right panel). Since speed was identical between the two varied speed conditions, but performance significantly differed, speed cannot account for the realistic physics benefits we find. As expected, the constant speed model always has constant speed; the two varied speed models have identical distributions; and these two speed distributions are closely matched to the realistic physics condition. 
PHYSICS IN TRACKING

Figure 5 shows that objects in the two varied speed conditions collide with one another more frequently than those in the constant speed and realistic physics conditions. If objects in a condition with more collisions are harder to track, tracking performance should be lower in the two varied speed conditions compared to either constant speed or realistic physics conditions, inconsistent with what we observe.

Overall, the spatial profiles of the four conditions are almost identical, while the temporal profiles of the two varied speed and the realistic physics conditions are very similar. Taking the results in Experiments 1 and 2 and the cumulative distribution analysis together suggests that the superior tracking ability in the "varied speed, correct angle" and the realistic physics conditions is unlikely due to the difference in spatial or temporal properties of the motion. The collision profiles reveal that the two varied speed conditions have more object collisions compared to the other two conditions. Referring to the behavioral results, however, the two conditions have very different accuracy patterns. Similarly, the constant speed and the realistic physics conditions have similar collision profiles, but behavioral performance of the realistic physics condition was consistently superior in both experiments. This suggests that the frequency of collisions alone has poor predictive power of the behavioral results. 


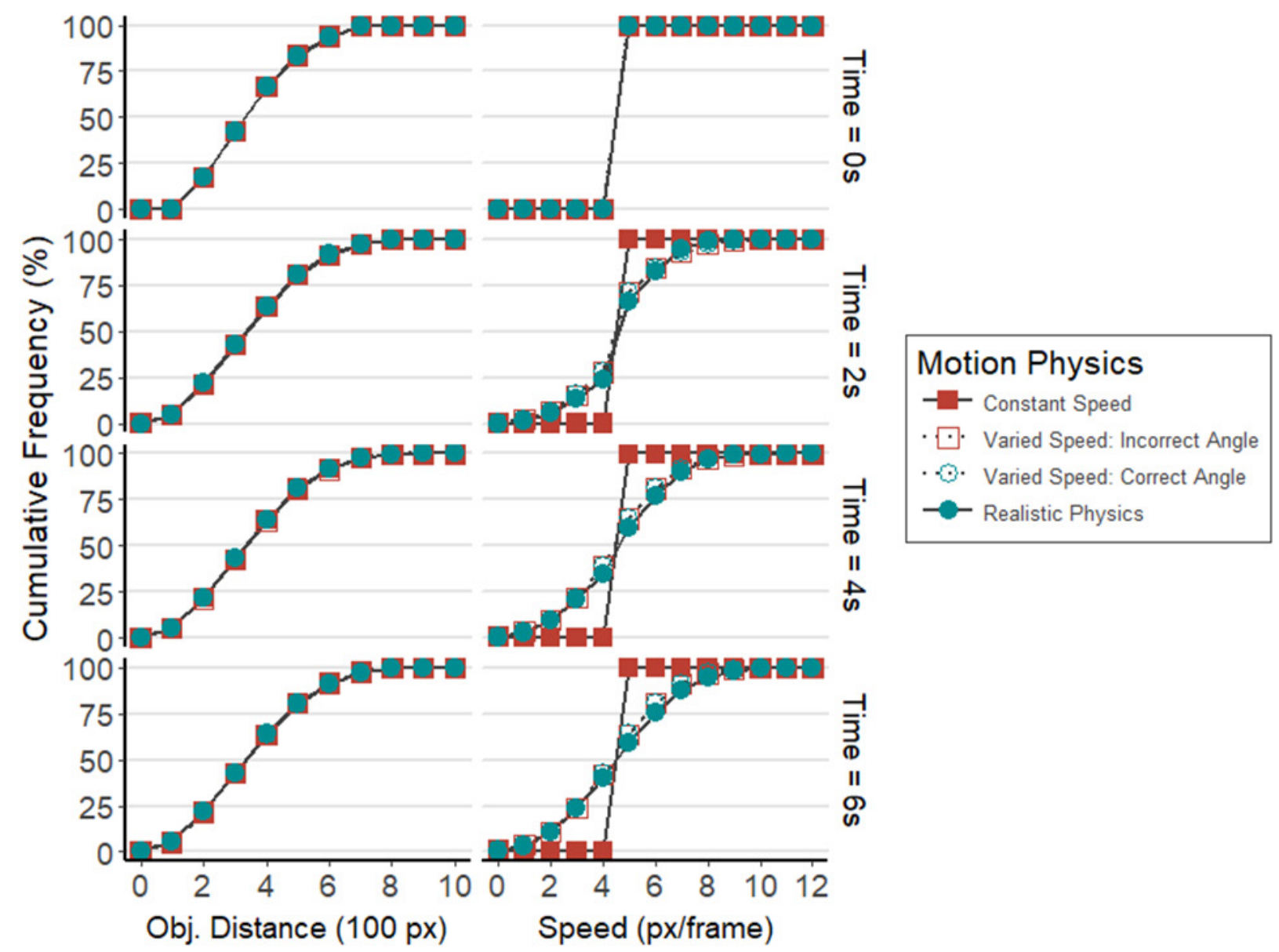

Figure 4. Spatial (left panel) and temporal (right panel) profiles of the conditions. Both spacing and speed are highly similar across conditions except in the way we explicitly manipulated (e.g., constant vs. variable speed), suggesting that differences in performance cannot be straightforwardly explained by these low-level properties. 


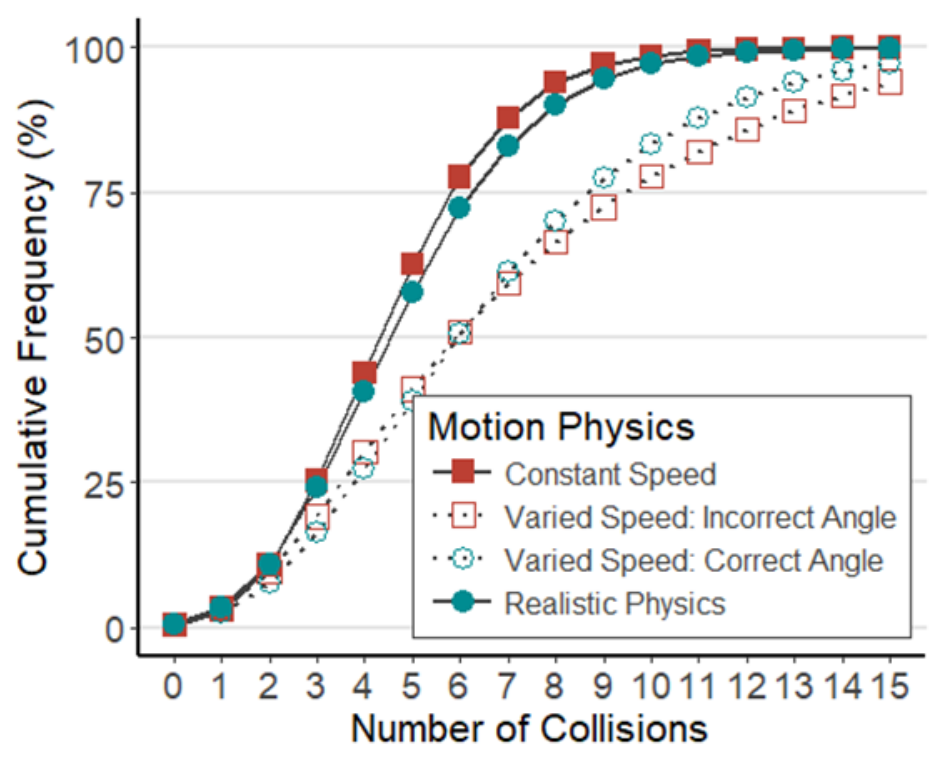

Figure 5. Collision profiles of the four conditions. The profiles of constant speed and realistic physics models are similar, but participants performed worse in the former condition than the latter. The profiles of the two varied speed conditions are also very similar, but participants performed better in the "varied speed, correct angle" condition. Again, this suggests that the frequency of collisions is not a good predictor of performance.

Note that these simulations consider objects and object distances, rather than collisions per se.

Thus, they do not rule out certain classes of low-level correlates of the physics conditions, especially ones that occur as an interaction between the two items at the moment of the collision. Since the conditions have different collision dynamics, there are in fact reliable low-level correlates of this that occur at the moment of the collision (e.g., the differential speeds between the two objects are greater in the realistic physics, by necessity). However, they do provide evidence that simple factors like the frequency of collisions alone or the speed of the objects alone do not likely account for our results. 
PHYSICS IN TRACKING

\section{Simulation 2: Difference in reflection angles between Realistic Physics and Varied}

\section{Speed, Incorrect Angle Models}

Experiment 2 shows that participants performed worse in the "Varied Speed, Incorrect Angle" condition compared to the realistic physics model or the "Varied Speed, Correct Angle" condition. We took that as evidence that people are sensitive to the reflection angle of an object upon collision.

Given the minor change that results in a major change in performance - and given that viewing these videos reveals they look rather natural - we believed it was important to investigate how the angles in this condition vary from what would be expected according to realistic physics. Thus, we ran a simulation of the "Varied Speed, Incorrect Angle" condition to quantify the deviations of the incorrect angles from the expected angles according to a realistic physics model (angle deviation). Extremely small angle deviations would argue against our hypothesis that the decrease in performance in the varied speed condition could be attributed to the incorrect angles.

\section{Method}

The simulation was similar to Simulation 1. Four hundred trials in the "Varied Speed, Incorrect Angle" condition were simulated. When two objects collided, the reflection angles of both objects were recorded. At the time of collision, we also simulated the reflection angles as if the objects followed a realistic physics model. The difference between the motion performed and the simulated angle forms an angle deviation score. The scores are aggregated in Figure 6.

\section{Results}

The simulation results show that there are clear deviations between the reflection angles in the "Varied Speed, Incorrect Angle" condition and a simulated realistic physics model. The distribution has a mean 
of $-0.41^{\circ}$ with a circular standard deviation of $69.71^{\circ}$. Thus, some angle deviations were quite substantial. This suggests that incorrect reflection angles are a plausible candidate to explain the difference in performance between the two Varied Speed conditions. In fact, it may be that the large deviations are systematically responsible for impaired performance in the two conditions with incorrect reflection angles (see Simulation 3). This is consistent with the possibility that we lose track of some objects only when the reflection angles of two colliding objects deviate substantially from the ones we would predict based on our intuitive understanding of object collision physics. Indeed, small deviations are unlikely to be noticeable given perceptual noise in our initial estimates of the objects' trajectories (Simulation 3).

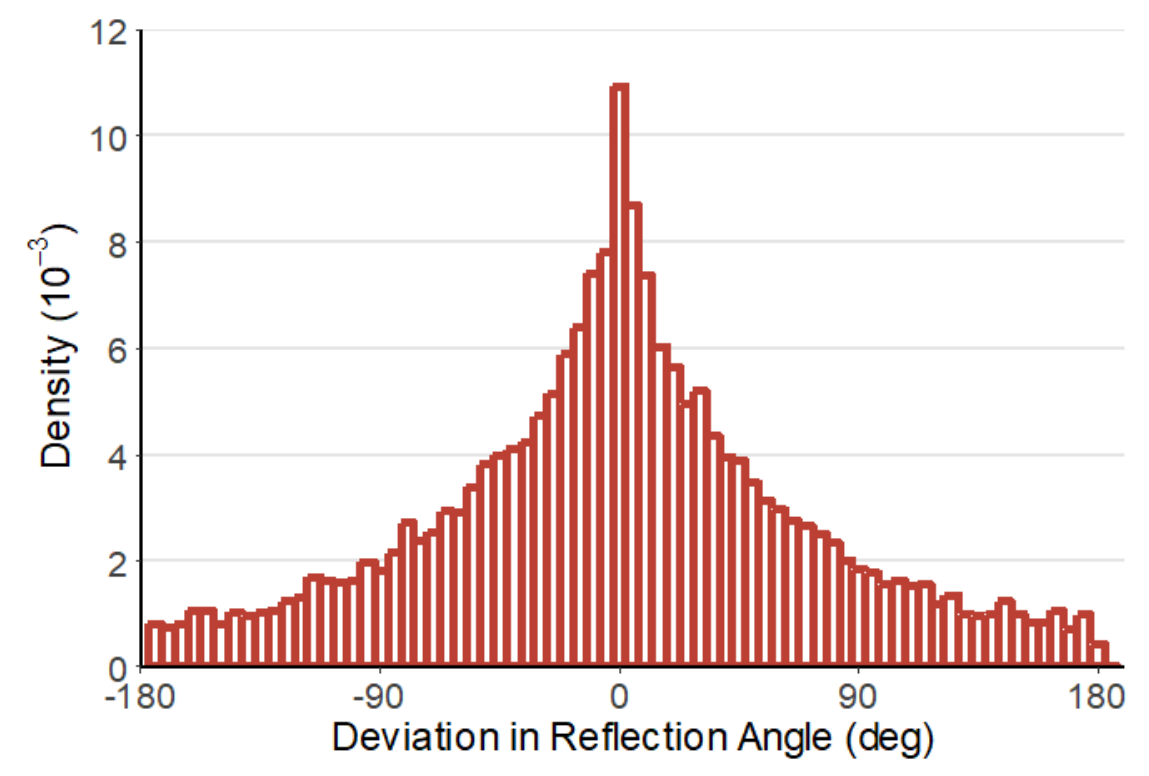

Figure 6. Deviation between the angle of reflection predicted by the realistic physics model and the angles in the "Varied Speed, Incorrect Angle" condition.

\section{Simulation 3: Effects of Perceptual Noise on Object Trajectory Estimation}

Experiments 1 and 2 both suggest that people track objects more accurately in situations where objects follow a Newtonian model of object collisions. In particular, observers seem to benefit when the object 
PHYSICS IN TRACKING

speeds are not constant, and having the correct reflection angle after collision seems to promote successful tracking. When objects collide with each other in the "Varied Speed: Incorrect Angle" condition, the reflection angles are calculated with reference to the constant speed model. As we saw in Simulation 2 even though the reflection angles deviate from those predicted by a Newtonian model, they are not entirely incorrect - the amount they deviate from the correct angle are highly variable. How these deviations affect performance is not entirely clear. On the one hand, people may be very sensitive to Newtonian physics, such that small deviations in reflection angles / speeds strongly compromise tracking performance. On the other hand, there might be intrinsic noise in the perceptual system, which in turn affects people's abilities to extrapolate post-collision object trajectories. This is how the existing literature on intuitive physics conceives of other more explicit cases of object physics judgment (“Noisy Newton” -- e.g., Sanborn, Mansinghka, \& Griffiths, 2013) - errors arise not from a flawed physics engine, but due to intrinsic perceptual noise in the estimation of the objects positions and trajectories. In this latter case, people would be accustomed to imprecise extrapolations of postcollision trajectories and thus would likely be tolerant to small deviations during tracking, since extrapolations are never precise in the first place. We would expect a decrease in performance only when a large deviation occurs. To examine this issue, we (1) performed another simulation, and (2) ran Experiments 3 and 4, which examine performance under conditions with smaller deviations in angle. Why might people be sensitive to only large angle deviations - and what counts as a large deviation? Perceptual noise in motion properties is inevitable. As a consequence, even if a perfect mental physics simulator is in place, a range of possible reflection angles and post-collision speeds is to be expected (as in Hamrick et al. 2011). Thus, to explore how perceptual noise affects post-collision motion trajectory, we ran a two-part simulation to examine whether noise in pre-collision speed or the angle has a larger influence on the post-collision trajectory of the objects, and the ranges of expected post-collision angles and speeds assuming small, medium, or large noise in perception of pre-collision 
PHYSICS IN TRACKING

angles and speeds. In the first part of the simulation, we insert jitter into the pre-collision speed and angle separately, as a model of perceptual noise, and consider only two objects in an isolated environment to allow clear visualizations of how such jitter impacts object trajectories. In the second part of the simulation, we vary both factors concurrently using the actual trajectories we utilized in our behavioral experiments and ask how large a set of deviations we would expect people to be tolerant based on reasonable estimates of perceptual noise they might have for each pre-collision object's trajectory.

If a variety of post-collision angles and/or speeds are possible assuming even a small amount of perceptual noise in the pre-collision objects' estimated speed and angles, we would expect people to discount the reliability of post-collision trajectories. In particular, we expect people would tolerate variability in the post-collision estimations at least as large as they would regularly expect from their perceptual precision. Thus, a wide range of post-collision angles and/or speeds with small or moderate noise in pre-collision speed or angle would lend support the idea that it may be that participants are sensitive to collision physics, but that most deviations go unnoticed - with only a subset of large deviations in angle leading to the impairment in the constant speed condition.

\section{Part 1 Method}

The simulation reported in this section makes use of the realistic physics simulator we utilized in Experiments 1 and 2. We begin - in Part 1- with particular, easy to visualize situations to give specific intuitions before considering the more general case (in Part 2). First, in Part 1, to aid visualization of object interactions, we only consider two interacting objects. One of the objects (Object 1) had a fixed pre-collision speed (at $10 \mathrm{px} /$ frame) and direction (moving to the right, i.e., $0^{\circ}$ from abscissa). The point of contact when the two objects collide was also fixed. In all three situations below, the only variables we independently manipulated were the speed and angle of incidence of the other object (Object 2). We consider these situations in detail as they are typical of collisions in the experiment. 
PHYSICS IN TRACKING

In Situation 1, Object 2's angle of incidence was $135^{\circ}$, when we varied the pre-collision speed from 0 to $20 \mathrm{px} /$ frame. That is, Object 2 had a speed that was within $\pm 100 \%$ of that of Object 1 . In Situation 2, its angle of incidence was $115^{\circ}$, when we again varied the pre-collision speed from 0 to 20 pixels per frame. Situations 1 and 2 give us an idea of how perceptual noise in speed affects predictions of post-collision trajectories. In Situation 3, Object 2's speed was fixed at $10 \mathrm{px} /$ frame, while varying the angle of incidence from $115^{\circ}$ up to $155^{\circ}$. Situation 3 shows us the effect of perceptual noise of angle of incidence on post-collision object trajectories.

In Figure 7, we show the pre-collision vectors of the Objects 1 and 2 in red and blue, respectively, and their post-collision trajectories in gray. Variations of the gray lines denote the influence of pre-collision speed (Situations 1 and 2), and angle of incidence (Situation 3) on the post-collision object trajectories.

\section{Part 1 Results}

Figure 7 shows the results of the simulations. Figures $7 \mathrm{a}$ and $7 \mathrm{~b}$ show how perceptual noise in precollision speed of an object leads to errors in predicting the post-collision speeds and moving directions of the two colliding objects. Figure 7c shows that when the pre-colliding speeds of the two objects were equal, perceptual noise in an object's angle of incidence has very little effect on the post-collision trajectory of the other object.

Thus, in these particular simulations, we assume people have a perfect physics simulator but a reasonable amount of perceptual noise. In these scenarios, we can infer that perceptual noise in precollision speed of an object has a larger influence on prediction of post-collision trajectory of colliding objects, compared to perceptual noise in pre-collision angle. As a consequence, if these scenarios are representative, people should be more tolerant to imprecise estimations of post-collision trajectories if they have perceptually noise estimates of pre-collision speed, compared to pre-collision angle. 
PHYSICS IN TRACKING

Part 1 represents a highly controlled environment in that either the pre-collision speed or direction of one object was manipulated to allow visualization (Figure 7). Part 2 aimed at understanding the contributions of both factors in a more naturalistic environment, when we allowed both precolliding speed and angle to vary. 
a

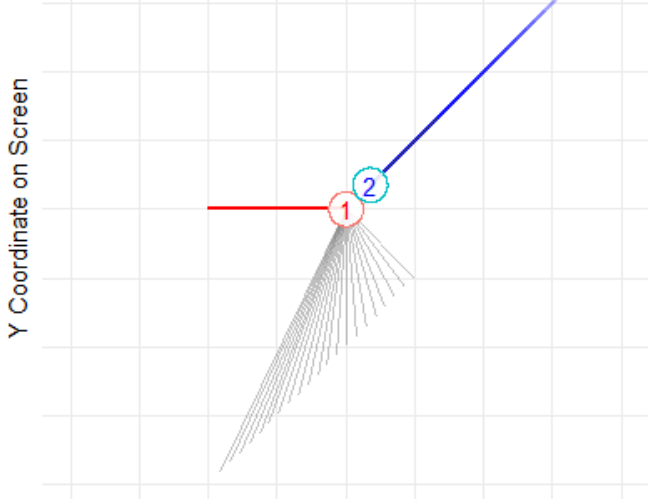

X Coordinate on Screen
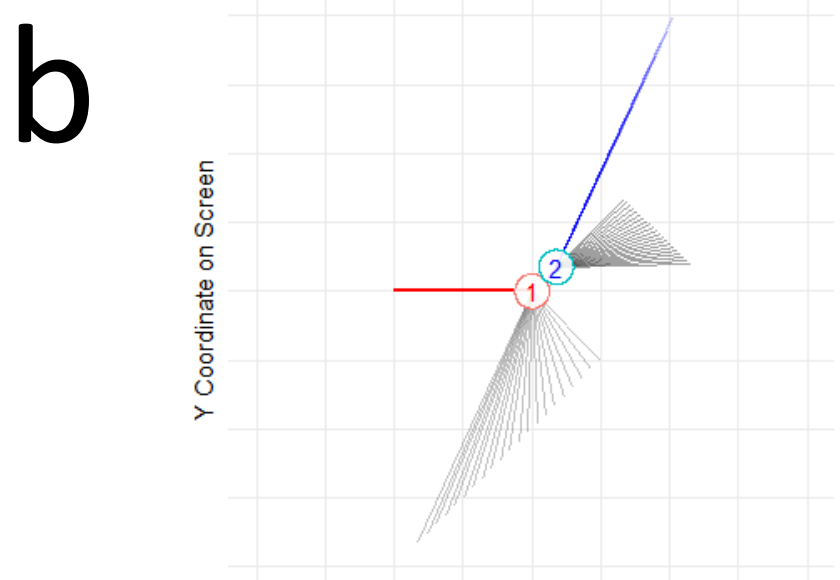

X Coordinate on Screen

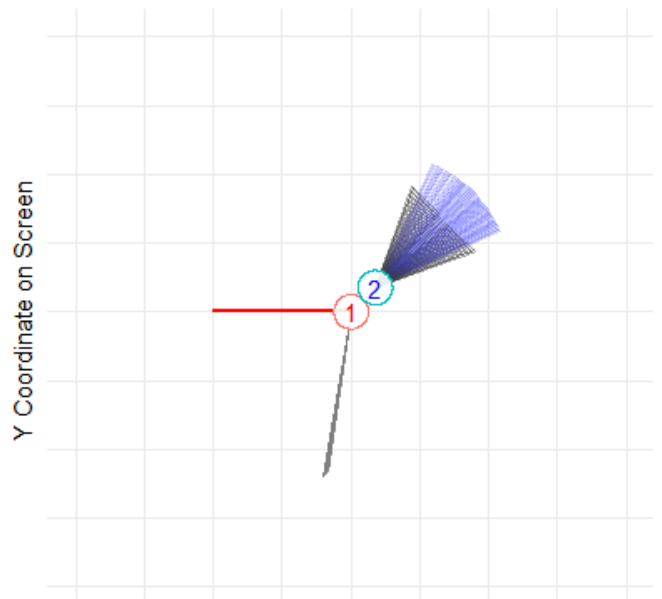

X Coordinate on Screen
Figure 7. Simulations of perceptual noise of pre-collision speed and angle on postcollision trajectory. Object 1's pre-collision speed and angle of incidence were fixed in all the situations. It was travelling at a speed of $10 \mathrm{px} /$ frame, moving to the right. The length of the lines represents the distance an object travels in 1 second, if we assume each grid represents 5 pixels. (a) Object 2's angle of incidence was $135^{\circ}$, and the speeds ranged from 0 to 20 pixels per frame. Object 2 followed the same path in a reversed direction after collision in all the cases, while Object 1's postcollision speed and direction varied a lot. It shows that perceptual noise in Object 2's pre-collision speed can lead to uncertainty in predicting Object 1's post-collision trajectory.

(b) Object 2 collided with Object 1 at $115^{\circ}$ at different speeds. There were more variations in Object 2's post-collision speed, compared to the situations in (a). It shows that perceptual noise in Object 2's pre-collision speed can lead to uncertainty in predicting its own post-collision trajectory, in addition to variations in Object 1's trajectory.

(c) Object 2's pre-collision speed was fixed at $10 \mathrm{px} /$ frame, while the angle of incidence varied from $115^{\circ}$ to $155^{\circ}$. The manipulation had very little impact on Object 1's post-collision trajectory. It shows that perceptual noise in Object 2's pre-collision angle have little influence on the estimation of Object 1's post-collision trajectory, but it can greatly affect the predicted direction of Object 2's postcollision direction. 
PHYSICS IN TRACKING

\section{Part 2 Method}

We created 300 trials using the realistic physics model in Experiments 1 and 2. As in the behavioral experiments, each object was initially randomly positioned within a rectangular window, with a starting speed of $5 \mathrm{px} /$ frame and a randomly determined starting direction. The objects traveled along a linear trajectory until they hit the border of the window. They also reflected from other objects when two of them came into contact. When two objects collided, we recorded each object's pre-collision and postcollision speeds and angles. In addition, for each collision, we added small, medium, or large jitter to the pre-collision speeds and angles, and observed how these deviations affected the simulated post-collision trajectories without actually changing the trajectories. Specifically, we added or subtracted 1, 2, or 3 $\mathrm{px} /$ frame to the pre-collision speed, and $5^{\circ}, 10^{\circ}$, or $15^{\circ}$ to the pre-collision angles. We took these to reflect small, medium and large amounts of plausible perceptual noise. The graphs in Figure 8 show the simulated post-collision speeds (i.e., speed deviations) and angles (i.e., angle deviations) relative to those predicted by the realistic physics model without noise. The distributions indicate how postcollision trajectories are affected by different levels of perceptual noise in pre-collision speeds and angles.

\section{Part 2 Results}

Of the 300 simulated trials, 28 had trajectories that would have caused us to exclude them for usage in the behavioral experiment (e.g., objects sticking together). Thus, we excluded these and considered the remaining 272 trials, which captured around 150,000 collisions and close to 2.7 million simulated points. Speed deviations and angle deviations were separately plotted in the two panels in Figure 8.

In general, perceptual noise - instantiated as simulated jitter - has a larger effect on the postcollision speeds, compared to the post-collision angles. In the most extreme case, the speed deviations span beyond \pm 5 pixels / frame, more than the initial speed of the objects. 
PHYSICS IN TRACKING

Comparing across columns in both graphs, the distributions become increasingly wider. This is consistent with the idea that adding jitter to the pre-collision speeds makes post-collision speeds and angles more unpredictable. On the other hand, comparing across rows, the distributions only change slightly. Adding jitter to the pre-collision angles has little effect on the post-collision trajectories, for both speed and angle. Therefore, trajectory changes due to perceptual noise in pre-collision angle is minimal.

Note that even in the most extreme cases that we simulated, the angle deviations were much smaller than those in the Simulation 2 section, when we compared the deviations between the angles of reflection predicted by the realistic physics and "varied speed, incorrect angle" conditions. Thus, even with reasonably large pre-collision perceptual noise in both angle and speed, participants should be pretty confident in the post-collision angle (within a range of $\sim 90^{\circ}$ ), although much less certain about the post-collision speed. Thus, comparing this with Simulation 2 suggests that a substantial number of collisions in the "varied speed, incorrect angle" condition had reflection angles so unnatural $\left(>90^{\circ}\right)$ even after taking very large amounts of perceptual noise into account, these angles might be noticeably incorrect for any observer who is making use of intuitive physical assumptions about object motion. However, a large number of the incorrect collisions - those less than $90^{\circ}$ and certainly those less than $60^{\circ}-$ would be perfectly consistent with intuitive physical reasoning, assuming noisy perceptual inputs. 
PHYSICS IN TRACKING
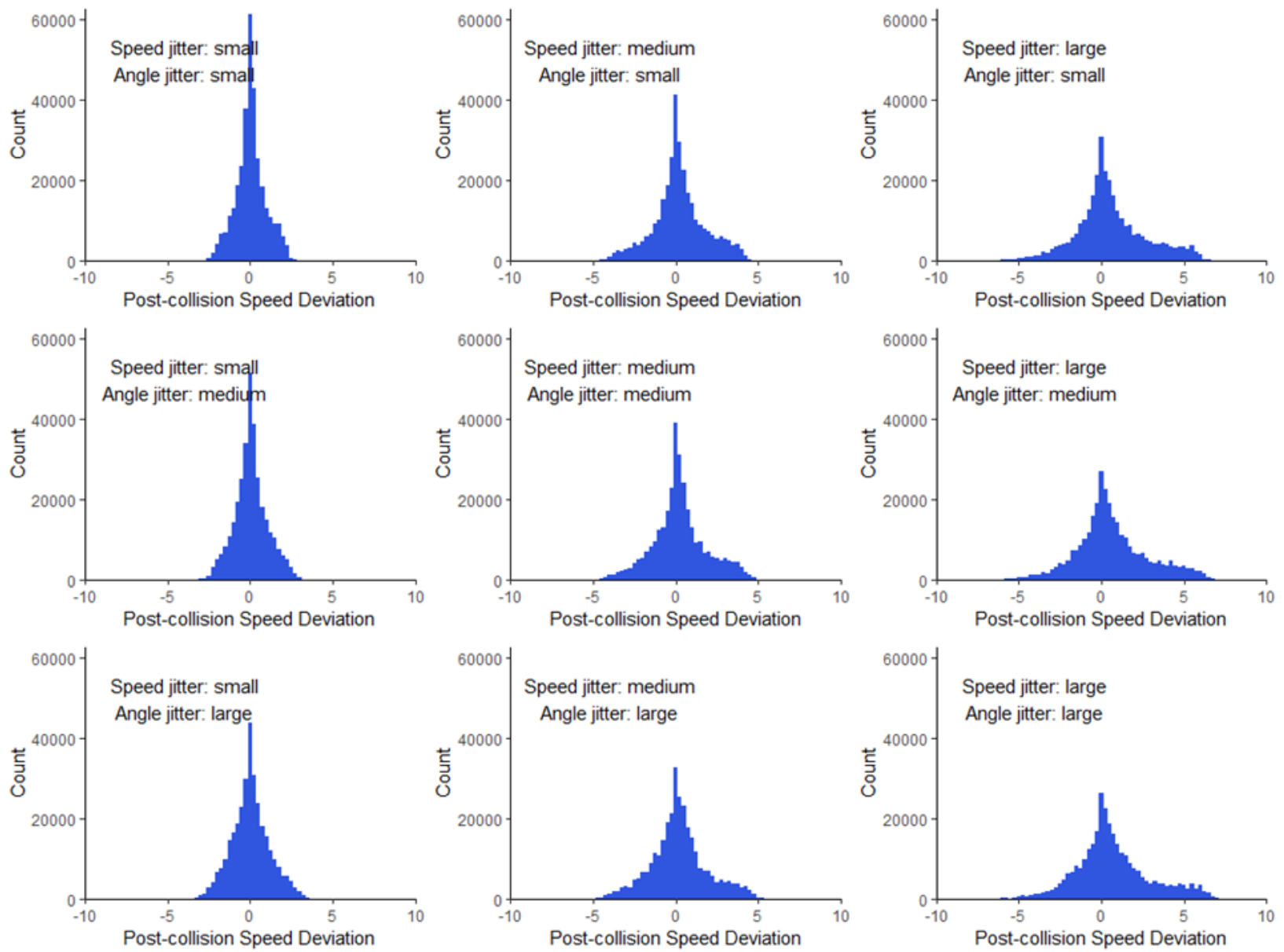

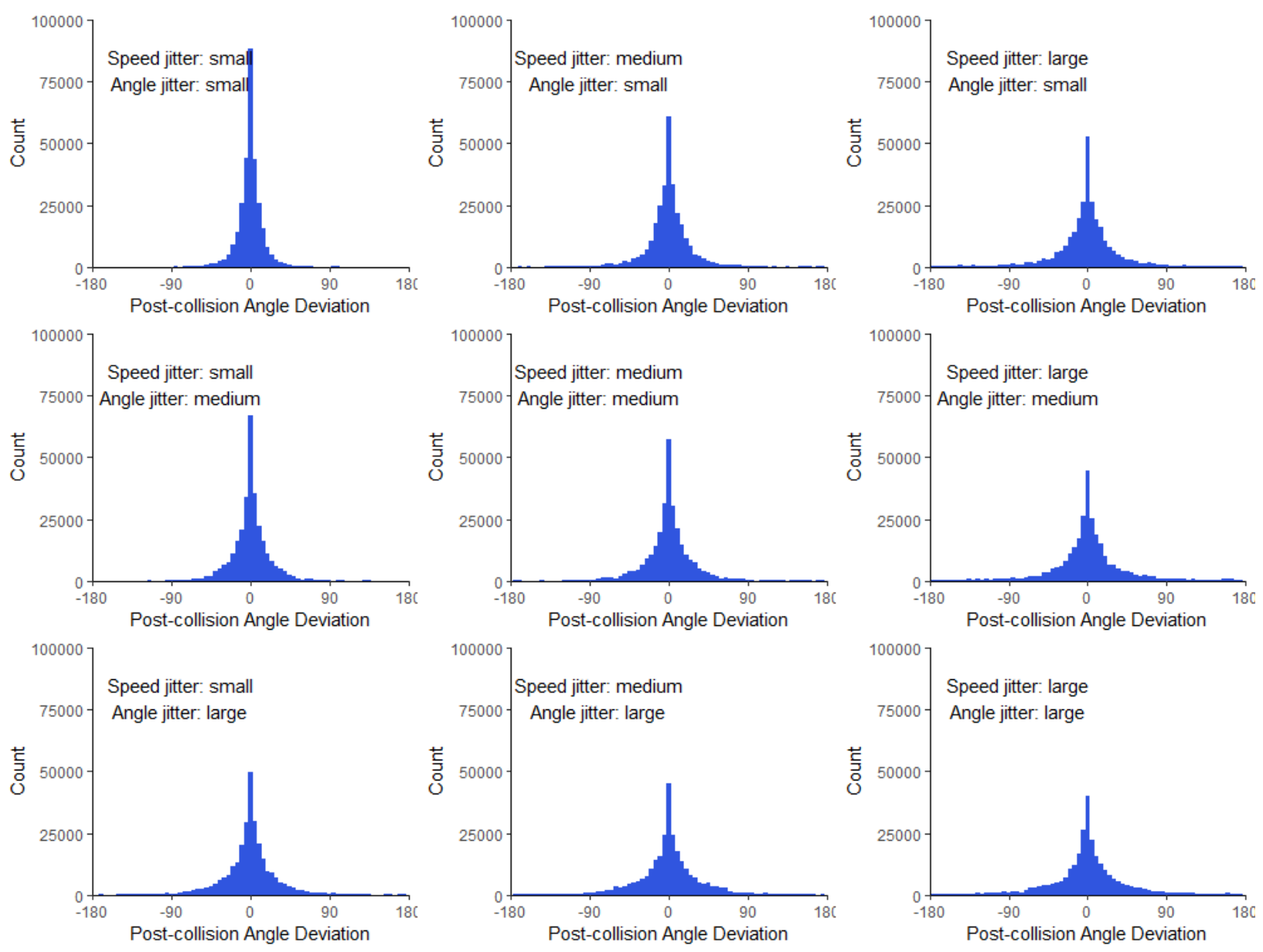

Figure 8. With small, medium, and large degrees of perceptual error in the pre-collision objects, we can expect the estimation of post-collision speed to deviate from the Newtonian physics model by up to \pm 5 pixels / frame (top panel), or $\pm 90^{\circ}$ (bottom panel). The post-collision angle deviations are much smaller in these simulations than in Simulation 2 section, indicating that a number of collisions in the "Varied Speed, Incorrect Angle" condition were unnatural even factoring in perceptual noise.

\section{Discussion}

We find that an extremely wide variety of post-collision speeds are possible assuming even a small amount of perceptual noise in the pre-collision objects' estimated speed. In general, a larger precollision speed jitter leads to more unpredictable post-collision speed. In contrast, perceptual noise in pre-collision speed and angle has a relatively minor effect on the estimation of post-collision angle. In our simulations, most of the deviations in post-collision angles fell within $\pm 90^{\circ}$ regardless of the level of 
PHYSICS IN TRACKING

jitter. The distributions are very different from that in Simulation 2. This further supports the idea that the large angle deviations may be the source of impairment in Experiment 2's constant speed and "varied speed, incorrect angle" conditions, as the level of deviations in those conditions went beyond what would be expected after incorporating perceptual noise. That is, even with reasonably large precollision perceptual noise in both angle and speed, participants should be pretty confident in the postcollision angle within a range of $\sim 60^{\circ}-90^{\circ}$, although much less certain about the post-collision speed. This could explain why participants are relatively unaffected by post-collision speed jittering, so long as this speed varies; and would suggest that a substantial number of collisions in the "varied speed, incorrect angle" condition had unnatural enough reflection angles $\left(>90^{\circ}\right)$ even after taking perceptual noise into account to impair performance. Overall, the simulations are consistent with the idea that only large angle deviations should be outside what participants expect from noisy Newtonian simulations, and that almost no post-collision speeds should be outside what is expected in a noisy Newtonian framework.

Importantly, however, these simulations do not argue that participants ignore the speed of objects. Even though the data from the experiments seem consistent with a larger role for post-collision direction than post-collision speed, the simulations show that in order to estimate the post-collision direction with any reasonable good precision, participants need an awareness of the pre-collision speed. Otherwise, the reflection direction is essentially unconstrained. Thus, the fact that post-collision angles affect performance requires that participants be using pre-collision angles and pre-collision speeds to calculate post-collision trajectories. Thus, in a framework broadly consistent with an accurate physics engine dealing with noisy perceptual data (Sanborn, Mansinghka, \& Griffiths, 2013), these simulations suggest an explanation for why multiple object tracking studies have not always found evidence for extrapolation of trajectories and other indexes of physical reasoning. If people have a large amount of uncertainty even in usual conditions (e.g., Howe \& Holcombe, 2012), the ability to extrapolate object 
PHYSICS IN TRACKING

trajectories after collisions is highly constrained by perceptual noise (e.g., Zhong et al. 2014). Thus, these simulations suggest that our studies provide evidence for the benefit of realistic physics largely via the use of more deviant angles and more variable speeds, and that participants should therefore not be sensitive to small angle deviations alone because these would be within the expected range given perceptual noise.

\section{Experiment 3: Performance with Jittered Angle}

The simulations above show that noise in perceiving pre-collision object trajectories makes it harder to predict the objects' post-collision speeds. On the contrary, the effect of perceptual noise on pre-collision angle is smaller, but still substantial.

In the first two experiments, we also showed that people are sensitive to some physics properties of motion, as their tracking performance was higher when some realistic physics properties were built into the motion. Together, these experiments show that people's tracking performance benefits from realistic physics properties, yet they tolerate small deviations from realistic physics. Randomizing the objects' speeds after post-collision has little effect on tracking performance, as long as the speed is not entirely unrealistic like in the constant speed condition. The same seems to apply to post-collision reflection angle. Performance was the best when reflection angles follow Newtonian physics, and was the worst when the reflection angles were allowed to deviate greatly from what would be predicted from the Newtonian physics model. It is unclear whether a consistent, but smaller deviation of post-collision direction would lead to a decrease in tracking performance. The noisy Newtonian framework suggests that if the angle deviations were within the range expected by perceptual noise they may not impair performance. Instead, only very large deviations like those used in Experiments 1 and 2 should impair performance. 
PHYSICS IN TRACKING

Thus, in Experiment 3, in addition to the realistic physics condition, we created two conditions where post-collision reflection angles always deviated from what would be expected from a Newtonian physics model by either $\pm 40^{\circ}$ or $\pm 80^{\circ}$ within a given trial. The deviations are of a small to medium size according to our analysis in Simulation 3. We intended to create a condition with a larger consistent deviation, but doing so also introduced artifacts in the motion such that they became entirely unrealistic when it occurred for every collision. Compared to performance in the realistic physics condition, we expected at most a small decrease in these "jittered angle" conditions if it is true that only large deviations - those that are unlikely even with significant perceptual noise in the judgment of the angle/speed of the pre-collision objects - are responsible for the impaired performance in the incorrect angle conditions.

\section{Method}

\section{Design}

In this experiment, participants were asked to track for four targets in each trial. We manipulated postcollision angle with a within-subjects design. In one-third of the trials, the object motion followed that of a realistic physics model, as in that in Experiments 1 and 2. In the other one-third of the trials, whenever two objects collided, the reflection speed and angle of an object were first calculated using a realistic physics model, and the angle was then jittered by around $40^{\circ}$ clockwise or counter-clockwise. For the rest of the trials, the post-collision angles were jittered by around $80^{\circ}$.

\section{Participants, Apparatus, Materials and Procedure}

Twenty-eight participants (23 female) were recruited from the UCSD Psychology Subject Pool, as described above. They had a mean age of 20.0 years at the time of participation. All participants took part in the study for partial course credits.

Participants went through 9 practice trials to familiarize themselves with the procedure, three for each jitter level. In the two jittered angle conditions, the post-collision angles of both objects were 
PHYSICS IN TRACKING

jittered around $40^{\circ}$ or $80^{\circ}$. The exact angle was randomly sampled from a uniform distribution with a range of $20^{\circ}$ of the respective mean. The jitter level within a trial was consistent. This created 3 jitter levels: none, small, and medium. Participants were asked to track 4 targets in each trial. They were asked to perform 4 test blocks, each with 30 trials. Trials with realistic physics or jittered post-collision angles were intermixed within each block, and the order of the trials were randomly determined.

\section{Results}

Accuracy was submitted to a one-way within-subject ANOVA, with the jitter level as the factor. In general, performance of the three conditions do not differ from each other, $F(2,54)=1.5, p=0.22$, Bayes Factor $=2.93$ in favor of the null hypothesis. As can be seen in Figure 9, accuracy in the realistic physics condition (84.2\%) is essentially the same as in the small jitter condition (84.5\%). Performance on medium jitter condition (83.3\%) differ from the two other conditions minimally. Therefore, we conclude that small to medium consistent post-collision angle jitters have minimal effects on tracking performance.

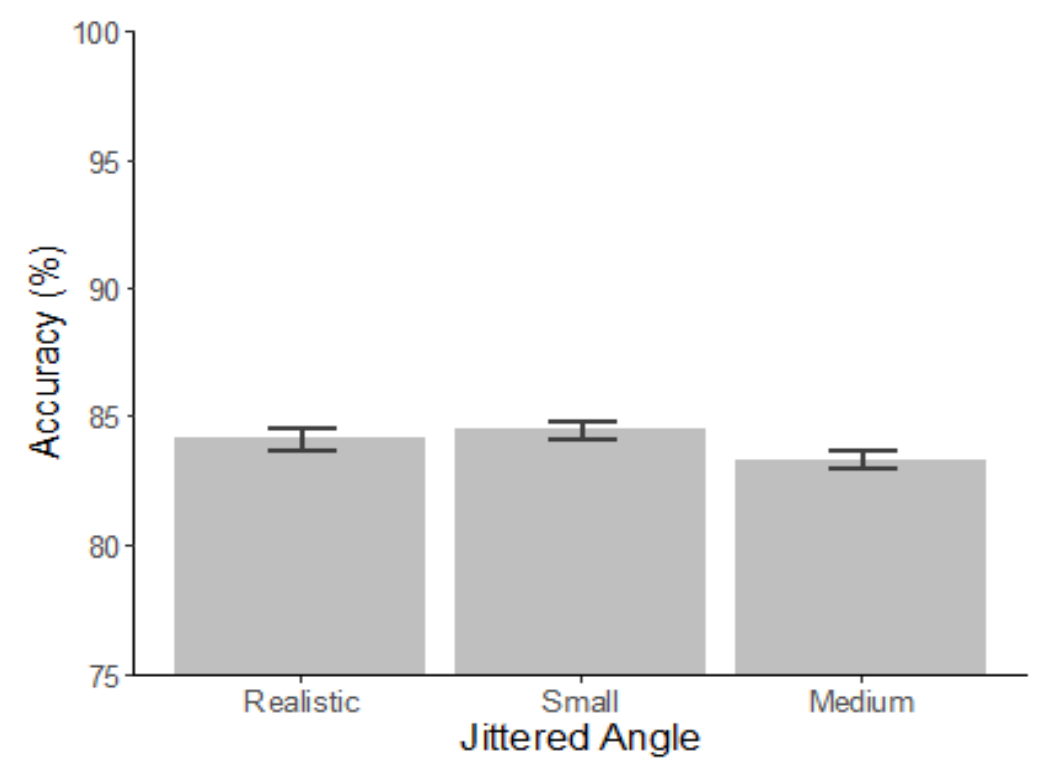

Figure 9. Accuracy data of Experiment 3. Post-collision angle jitters from a realistic physics model had little effect on tracking performance. This echoes the results in Simulation 3, which shows that people's estimation of post-collision angles are unlikely to be very precise due to perceptual noise. 
PHYSICS IN TRACKING

\section{Conclusion}

Experiment 3 provides behavioral evidence for the results in Simulation 3, supporting the idea that people are not very sensitive to precise post-collision motion directions within the range expected from noisy Newtonian simulations. The results strongly suggest that the lower tracking performance in the Constant Speed and "Varied Speed, Incorrect Angle" conditions in Experiment 2 was driven by occasional highly incorrect reflection angles in these conditions.

As mentioned in the Introduction, the constant speed model in Experiments 1 and 2 has strong regularities, which could have helped tracking - and which observers' reported they believed had helped their tracking. However, compared to the realistic physics condition, these strong regularities are not more helpful for tracking in the presence of other factors, but lead to occasional highly incorrect reflection angles as by-products.

This leads us to hypothesize whether a new constant speed model with correct reflection angles would allow people to perform at a level that is comparable to the realistic physics condition. In Experiment 4, we created such a new variant of the constant speed condition, in which the reflection angles are correct according to Newtonian physics (i.e., constant speed, correct angle).

If the occasional highly incorrect angles are the major source of error in the constant speed condition in Experiments 1 and 2, removing these incorrect angles should bring the performance up to a level comparable to the realistic physics condition. With their high regularities, there is a possibility that people could perform better in this new constant speed condition, compared to the realistic physics condition. 
PHYSICS IN TRACKING

\section{Experiment 4: Performance with Constant Speed, Correct Angle Condition}

\section{Method}

\section{Design}

As in Experiment 1a, we manipulated the object speed across subjects, such that each participant either encountered the entire experiment with object speed being constant or moving according to a realistic physics model. Participants were randomly assigned into one of the two conditions. In Experiments 1 and 2, when two objects collided in the constant speed condition, the motion directions of the objects exchanged. The objects therefore maintained a constant speed throughout the trial because they started off with the same speed. As a consequence, the reflection angles deviated from what would be expected from a Newtonian model.

MOT is an attentionally-demanding process., and there are probably multiple moments of uncertainty about the identity of the target items during the course of tracking. If such uncertainty took place at the exact moment when a target and a distractor collided, observers might have missed the events and hence failed to update the identities of the two objects. In Experiments 1 and 2, colliding objects in the constant speed condition took on the velocities of the other object (as their velocities simply exchanged, see Figure 1). Thus, if an observer missed the collision, the two colliding objects would look like they had continued following their existing paths (although the identities were swapped). On the other hand, in the realistic physics condition, both the speed and velocity of an object changed after a collision, so it is possible this would make the collision harder to miss. Hence, failure in identity updating might be more prevalent in the constant speed condition than the realistic physics condition in Experiments 1 and 2, due to the regularity of reflection angles.

In this current experiment, we created the constant speed condition by modifying the realistic physics model. When two objects collided, the speeds of the objects were reset to the initial speed. 
PHYSICS IN TRACKING

Therefore, when the object collided, they had the correct reflection angles that would be predicted by the Newtonian physics model, and they had a constant speed throughout the trials.

In each trial, participants either tracked 2 or 4 targets. In half of the trials, we imposed $45^{\circ}$ jitters on the post-collision angles, as in Experiment 3. These latter two factors were manipulated withinsubjects, and the order of the trials was randomized within each block. The trials with $45^{\circ}$ jitters served as an attempt to replicate our results in Experiment 3. If collisions in the constant speed condition were easier to miss in Experiments 1 and 2 because of the regularity of reflection angles, the difference between the two constant speed and realistic physics conditions would be greatly reduced or eliminated in this Experiment.

\section{Participants, Apparatus, Materials and Procedure}

As this was a between-subjects experiment, we ensured that at least 30 participants were recruited for each of the two conditions to satisfy the normality assumptions for the statistical tests. Seventy-six participants (54 female) were recruited from the UCSD Psychology Subject Pool, as described above. They had a mean age of 20.1 years at the time of participation. All participants took part in the study for partial course credits.

Participants were randomly assigned into the "constant speed, correct angle" or the realistic physics condition. The two conditions were identical except that the object speeds were reset to their initial speeds of $5 \mathrm{px} /$ frame upon collision. Therefore, the reflection angles in both conditions were correct according to Newtonian physics. Participants went through 8 practice trials to familiarize themselves with the procedure, and completed 4 test blocks of 32 trials each.

\section{Results}

Around $10 \%$ of all trials were removed due to highly unnatural motion behaviors; however, exclusion of the data does not change the conclusion of the experiment. The rest of the data was submitted to a $2 \mathrm{x}$ 
$2 \times 2$ ANOVA, with object speed as a between-subjects factor, and number of targets and jitter level as the within-subjects factors.

As shown in Figure 10, participants attained a higher accuracy when tracking two targets, compared to four targets, $F(1,74)=62.7, p<0.001$, Cohen's $d_{z}=0.48$. The performance between the "constant speed, correct angle" and realistic physics conditions did not reliably differ from each other, $F(1,74)=2.0, p=0.162$, Cohen's $d=0.34$. The Bayes Factor is 0.69, which does not provide strong support for either the null or the research hypothesis. None of the other comparisons nor interactions reach statistical significance, $\mathrm{F}<1$. The differences between the new constant speed and realistic physics models had been greatly reduced from Experiments 1 and 2, but difference in raw mean accuracy is still notable. Thus, it is unclear whether the effect found in Experiments 1 and 2 can be fully attributed to the failure to notice identity switches during object collisions.

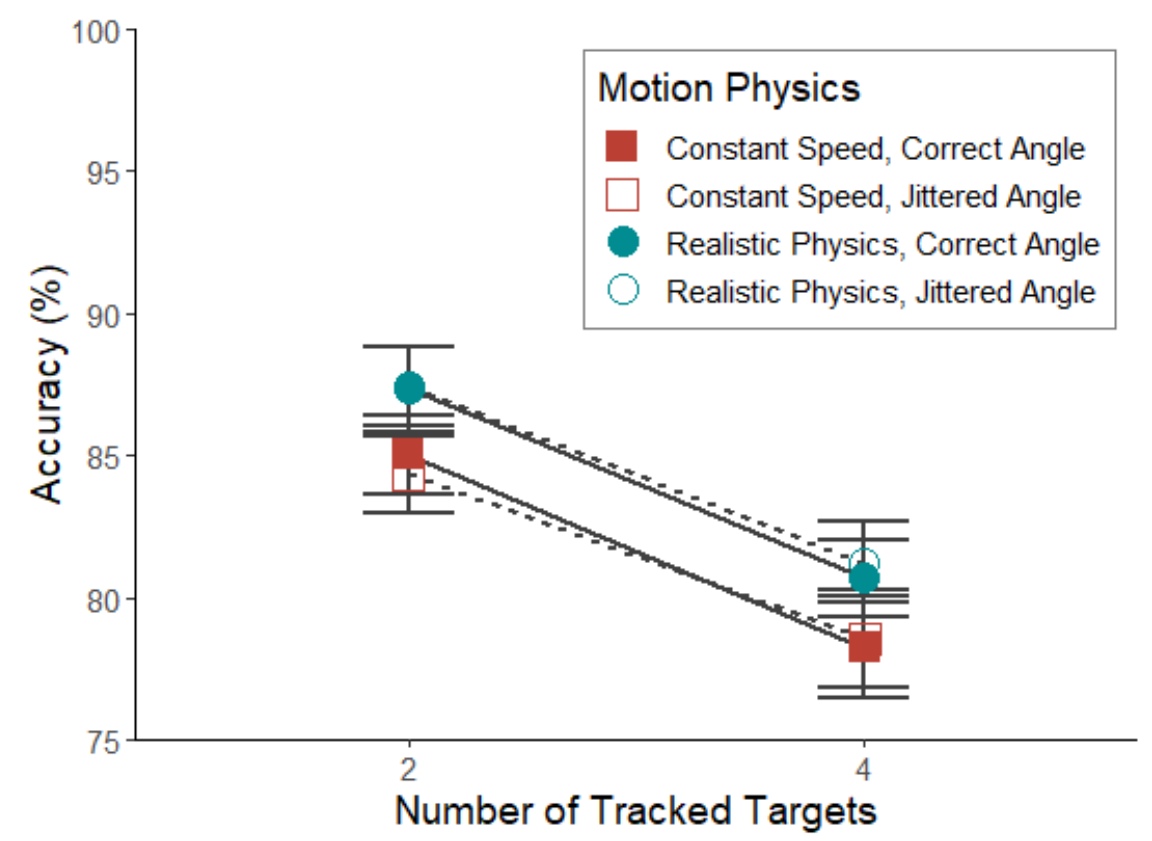

Figure 10. Accuracy data of Experiment 4. Jittering post-collision object angles does not affect tracking performance, as the transparent data points overlaps with the opaque ones. Allowing objects to vary in speed improves performance, although the effect is small and not statistically significant. 
PHYSICS IN TRACKING

\section{Conclusion}

A few observations in Experiment 4 are worth noting. First, jittering post-collision angles has virtually no effects on performance, mirroring the findings in Experiment 3. Second, the "constant speed, correct angle" group in the current experiment performed better than those in the "constant speed" (incorrect angle) condition in Experiment 1, compared to their corresponding realistic physics conditions. The effect size of the realistic physics advantage dropped from 0.88 in Experiment 1 to 0.34 in Experiment 4. This strongly suggests that people are sensitive to the post-collision reflection angles.

In Experiment 4, despite having correct post-collision reflection angles in both the constant speed and realistic physics condition, there is only a slight difference in performance. The effect ( $\approx 2.6 \%)$, measured by effect size, is small due to between groups variations and was not significant. However, it may be that this reflects some realistic physics advantage that arises from being able to utilize the variable object speeds.

\section{Comparison Across Experiments}

Throughout our 4 experiments, we have many within-experiment comparisons. In general, these comparisons point to an advantage for more realistic physics, and to an advantage for more realistic rather than less realistic variations (e.g., variable object speed vs. constant object speed). To summarize all of our manipulations, we plot the average accuracy of all the conditions when participants were asked to track 4 targets (Figure 11). We color-coded the manipulation of speed and angle for clarity, with more realistic versions having more saturated colors. In general, tracking performance was high when speed followed a realistic physics model. Randomizing the speed does not seem to affect performance, but having a constant speed model clearly harmed performance.

Regarding post-collision reflection angle, performance was high when the reflection angle followed a realistic physics model. When the angles were consistently jittered within a trial, to a small extent $\left( \pm 45^{\circ}\right)$, performance was unaffected. Performance suffers only in conditions when we allowed 
occasional very incorrect reflection angles. In general, the figure shows that across all experiments, there is a reliable trend for more realistic speeds and more realistic angles to improve performance.

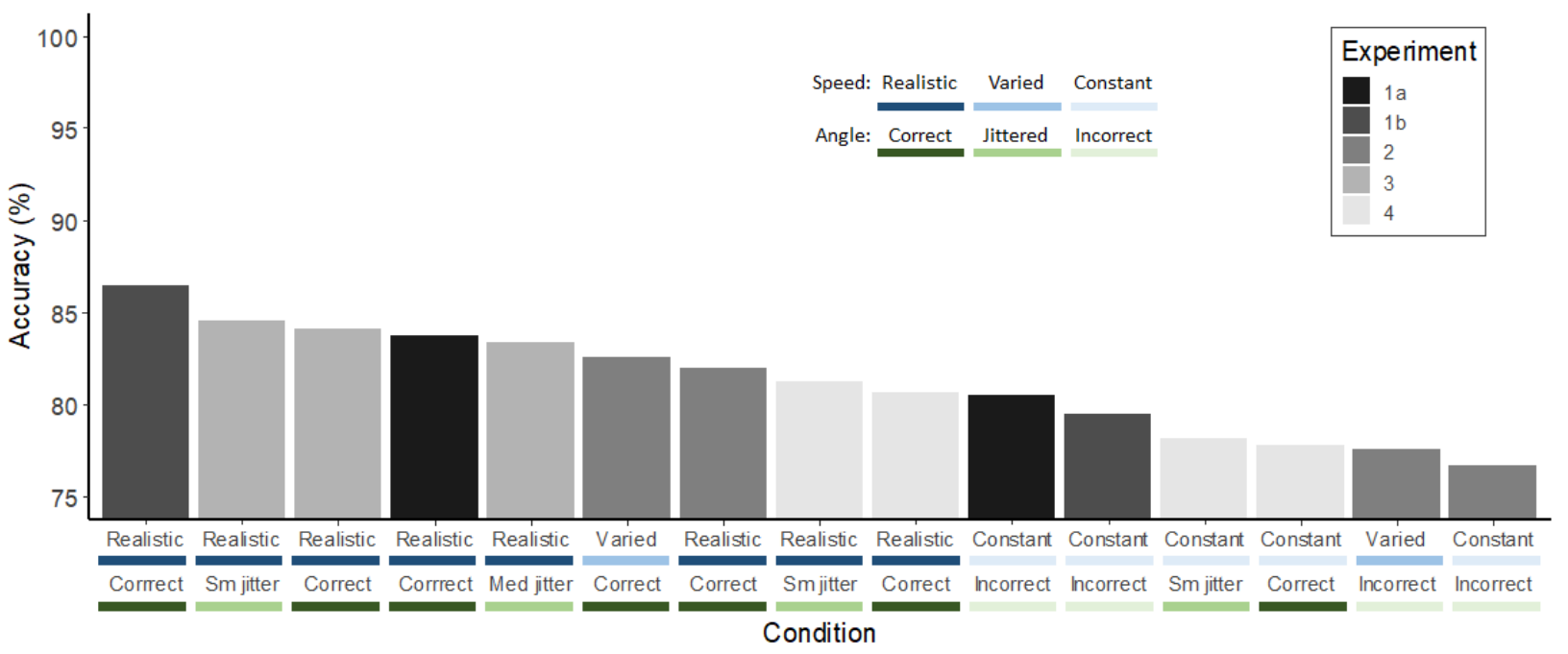

Figure 11. Average accuracy of the experiments on trials when participants tracked 4 targets. Performance was the best when the speed followed a realistic physics model, and the post-collision reflection angle followed the same realistic physics model. Performance across all experiments was generally lower with decreasing resemblance to the realistic physics model.

\section{General Discussion}

In four experiments, we show that people track moving objects more accurately when they move according to realistic physics compared to when they follow a constant speed model, although participants incorrectly believe that the simpler and more predictable motion in the constant speed model is easier. From our simulations, we conclude that the realistic physics benefits are unlikely to be the result of low-level motion properties such as object distance, speed, and frequency of object collisions. We find that people are far from perfect at making use of realistic physics: People are tolerant to small deviations of reflection angles and to variable but incorrect speeds. In our simulations, we show that this is likely due to intrinsic perceptual noise in the visual system, such that perfect prediction of object trajectory after collision is impossible - and speed is particularly difficult to correctly predict. Nevertheless, our simulations show that in order to be sensitive to post-collision angle deviations, 
PHYSICS IN TRACKING

participants must be somewhat sensitive to pre-collision angle and pre-collision speed. Thus, overall, we suggest that a 'Noisy Newton' framework for object tracking is plausible: people appear to be sensitive to large angle deviations in particular, but across all of our experiments participants show the best performance in the most realistic physics conditions.

These results renew our understanding of object tracking in the human mind. In particular, under attentional demanding conditions, people appear to be sensitive to several, but not all, important aspects of the laws of object motion according to Newtonian physics. People are sensitive to the speed of individual objects, and the reflection angle after object collisions. However, they do not seem to keep track of whether the objects' speed after collision is realistic, perhaps because such speeds are highly unpredictable if there is perceptual noise in the pre-collision objects' trajectories. Our results also indicated that previous research using the constant speed model underestimates people's true objecttracking abilities in a naturalistic environment by around 7\%. This is counter-intuitive if the brain's sensitivity to object physics is not considered: by having objects that are in many ways more individually unpredictable, people's tracking abilities are improved.

Why is correct angle seemingly of more importance than correct speed after a collision? One possibility is that this reflects genuine differences in people's expectation about the physics of object motion given the noise in their pre-collision estimates of speed and angle, as suggested by the simulation. However, it may also be that when participant's task is primarily to track objects - and keep targets separate from distractors - then in the critical window of the first few moments after the collision, the angle of the objects provides objectively more information about which object is which than the speed. That is, the angle may be faster to estimate and more diagnostic of which object is which because spatial location is easier to perceive than speed is to estimate, and because speed takes time to estimate whereas the angle is determined nearly right away (for related arguments, see Vul et al., 2009). 
PHYSICS IN TRACKING

An emerging view in cognitive psychology posits that people make use of a "physics engine" in the brain (Battaglia, Hamrick \& Tenenbaum, 2013; Hamrick, Battaglia, \& Tenenbaum, 2011). To make decisions, this internal "game engine" simulates possible physical outcomes. A decision is then made by comparing the relative likelihood of outcomes in the simulations. This physics engine view has been successful in explaining people's decisions about whether a tower of blocks will fall (Hamrick, Battaglia, \& Tenenbaum, 2011), whether a moving ball will fit into a slot (Hamrick, Smith, Griffiths, \& Vul, 2015), and the dynamics of liquid in the presence of obstacles (Bates, Yildirim, Tenenbaum, \& Battaglia, 2018), among other outcomes. An extension of this view, the noisy Newton framework, is able to bridge to previous findings in the literature on people's biased judgment (e.g., McCloskey, Caramazza, \& Green, 1980; McCloskey, Washburn, \& Felch, 1983; Kaiser, Proffitt, Whelan, \& Hecht, 1992; see also Sanborn, Mansinghka, Griffiths, 2013). To date, however, most research on this kind of intuitive physics has focused on physics reasoning tasks, largely those where people are explicitly directed to predict the outcome of a situation (either interactively or in the abstract: Smith, Battaglia, \& Vul, 2018), and in these situations it has been claimed that people make use of a rather cognitively expensive set of 3 to 7 physics engine simulations to make a judgment (Battaglia, Hamrick \& Tenenbaum, 2013). Our study raises the possibility that models of intuitive physics, at least for object motion patterns, are applied in more demanding situations like those encountered in real life, even when such knowledge must be applied very quickly in situations where multiple objects are constantly interacting with each other. Interestingly, in all of our experiments we found very comparable effects of object motion dynamics on both set size 2 and set size 4, rather than finding the effects diminished with increasing set size. This could mean they are somewhat automatic and would occur even under more cognitively demanding conditions and at higher set sizes, although this remains a question for future research.

One additional interesting result in our experiments is that people's subjective impression of difficulty did not track their objective performance. The simpler, more predictable constant speed 
PHYSICS IN TRACKING

displays led participants to believe they would perform best, even though they did not do so. One possible explanation for this is that because the objects in this condition are more confusable (they have no unique features, like the objects have unique speeds in the realistic physics condition), participants may be more likely to 'swap' objects (e.g., Drew et al. 2013) -- that is, they may believe they have continued to track the correct objects, despite having incorrectly assigned the objects' identities after a collision. Such swaps may impact performance without participants being aware of them. Future work could investigate this directly.

Broadly speaking, understanding how accurate and useful the people's intuitive physics sense is — and how accurately this sense of physics is used continuously in cognitively demanding situationshas values in many domains. For example, in science education, it helps teachers to close the gaps between laws of physics and students' intuition (e.g., McCloskey, Washburn, \& Felch, 1983). A logical extension of the present study would involve object tracking in 3-dimensional space. Previous research using both 2D and 3D stimuli suggests that effects found in 2D generally apply to 3D space as well (e.g., Liu, Austen, Booth, Fisher, Argue, Rempel, \& Enns, 2005; St. Clair et al, 2010). Understanding whether people track moving objects better in 3D space when they obey physical laws will allow the current effect to be extended to real life scenarios. It will also bridge the gap between the current research and previous efforts aiming to understand how people behave in an environment beyond artificial 2D displays (e.g., Meyerhoff, Huff, Papenmeier, Jahn, Schwan, 2011; Papenmeier, Meyerhoff, Brockhoff, Jahn, \& Huff, 2017). Future work on intuitive physics could also attempt to further close the gap between performance in demanding tasks like MOT and explicit judgments of physical situations. 
PHYSICS IN TRACKING

\section{Authors' notes}

The programs, data, and sample videos of the experimental conditions in the study are available at: https://osf.io/5hcs8

\section{Acknowledgements}

This research was generously supported by NSF CAREER Grant \#1653457 and NSF Grant \#1829434 to TFB. 


\section{References}

Alvarez, G. A., \& Cavanagh, P. (2005). Independent resources for attentional tracking in the left and right visual hemifields. Psychological science, 16(8), 637-643.

Bates, C. J., Yildirim, I., Tenenbaum, J. B., \& Battaglia, P. (2019). Modeling human intuitions about liquid flow with particle-based simulation. PLoS computational biology, 15(7), e1007210.

Battaglia, P. W., Hamrick, J. B., \& Tenenbaum, J. B. (2013). Simulation as an engine of physical scene understanding. Proceedings of the National Academy of Sciences, 201306572.

Carey, S., \& Xu, F. (2001). Infants' knowledge of objects: Beyond object files and object tracking. Cognition, 80(1-2), 179-213.

Cooke, J. R., ter Horst, A. C., van Beers, R. J., \& Medendorp, W. P. (2017). Effect of depth information on multiple-object tracking in three dimensions: A probabilistic perspective. PLoS Computational Biology, 13(7), e1005554.

Drew, T., Horowitz, T. S., \& Vogel, E. K. (2013). Swapping or dropping? Electrophysiological measures of difficulty during multiple object tracking. Cognition, 126(2), 213-223.

Eriksen, B. A., \& Eriksen, C. W. (1974). Effects of noise letters upon the identification of a target letter in a nonsearch task. Perception \& Psychophysics, 16(1), 143-149.

Fencsik, D. E., Klieger, S. B., \& Horowitz, T. S. (2007). The role of location and motion information in the tracking and recovery of moving objects. Perception \& Psychophysics, 69(4), 567-577.

Flombaum, J. I., Kundey, S. M., Santos, L. R., \& Scholl, B. J. (2004). Dynamic object individuation in rhesus macaques: A study of the tunnel effect. Psychological Science, 15(12), 795-800.

Franconeri, S. L., Jonathan, S. V., \& Scimeca, J. M. (2010). Tracking multiple objects is limited only by object spacing, not by speed, time, or capacity. Psychological Science, 21(7), 920-925.

Franconeri, S. L., Lin, J. Y., Enns, J. T., Pylyshyn, Z. W., \& Fisher, B. (2008). Evidence against a speed limit in multiple-object tracking. Psychonomic Bulletin \& Review, 15(4), 802-808.

Franconeri, S. L., Pylyshyn, Z. W., \& Scholl, B. J. (2012). A simple proximity heuristic allows tracking of multiple objects through occlusion. Attention, Perception, \& Psychophysics, 74(4), 691-702.

Gilden, D. L., \& Proffitt, D. R. (1994). Heuristic judgment of mass ratio in two-body collisions. Perception \& Psychophysics, 56(6), 708-720.

Hamrick, J. B., Battaglia, P. W., \& Tenenbaum, J. B. (2011). Internal physics models guide probabilistic judgments about object dynamics. In Proceedings of the 33rd Annual Conference of the Cognitive Science Society (pp. 1-6).

Hamrick, J. B., Smith, K. A., Griffiths, T. L., \& Vul, E. (2015). Think again? The amount of mental simulation tracks uncertainty in the outcome. In Proceedings of the 37th annual conference of the cognitive science society (pp. 1-6).

Holcombe, A. O., \& Chen, W. Y. (2013). Splitting attention reduces temporal resolution from $7 \mathrm{~Hz}$ for tracking one object to $<3 \mathrm{~Hz}$ when tracking three. Journal of Vision, 13(1), 12-12.

Horowitz, T. S., Birnkrant, R. S., Fencsik, D. E., Tran, L., \& Wolfe, J. M. (2006). How do we track invisible objects?. Psychonomic Bulletin \& Review, 13(3), 516-523.

Horowitz, T. S., \& Cohen, M. A. (2010). Direction information in multiple object tracking is limited by a graded resource. Attention, Perception, \& Psychophysics, 72(7), 1765-1775.

Howe, P. D., \& Holcombe, A. O. (2012). Motion information is sometimes used as an aid to the visual tracking of objects. Journal of Vision, 12(13), 10-10.

Howard, C. J., Masom, D., \& Holcombe, A. O. (2011). Position representations lag behind targets in multiple object tracking. Vision Research, 51(17), 1907-1919.

Intriligator, J., \& Cavanagh, P. (2001). The spatial resolution of visual attention. Cognitive Psychology, 43(3), 171-216. 
Iordanescu, L., Grabowecky, M., \& Suzuki, S. (2009). Demand-based dynamic distribution of attention and monitoring of velocities during multiple-object tracking. Journal of vision, 9(4), 1-1.

Kaiser, M. K., Proffitt, D. R., Whelan, S. M., \& Hecht, H. (1992). Influence of animation on dynamical judgments. Journal of experimental Psychology: Human Perception and Performance, 18(3), 669.

Keane, B. P., \& Pylyshyn, Z. W. (2006). Is motion extrapolation employed in multiple object tracking? Tracking as a low-level, non-predictive function. Cognitive Psychology, 52(4), 346-368.

Liu, G., Austen, E. L., Booth, K. S., Fisher, B. D., Argue, R., Rempel, M. I., \& Enns, J. T. (2005). Multipleobject tracking is based on scene, not retinal, coordinates. Journal of Experimental Psychology: Human Perception and Performance, 31(2), 235.

Meyerhoff, H. S., Huff, M., Papenmeier, F., Jahn, G., \& Schwan, S. (2011). Continuous visual cues trigger automatic spatial target updating in dynamic scenes. Cognition, 121(1), 73-82.

Meyerhoff, H. S., Papenmeier, F., \& Huff, M. (2013). Object-based integration of motion information during attentive tracking. Perception, 42(1), 119-121.

Meyerhoff, H. S., Papenmeier, F., \& Huff, M. (2017). Studying visual attention using the multiple object tracking paradigm: A tutorial review. Attention, Perception, \& Psychophysics, 79(5), 1255-1274.

Meyerhoff, H. S., Papenmeier, F., Jahn, G., \& Huff, M. (2016). Not FLEXible enough: Exploring the temporal dynamics of attentional reallocations with the multiple object tracking paradigm. Journal of Experimental Psychology: Human Perception and Performance, 42(6), 776.

McCloskey, M., Caramazza, A., \& Green, B. (1980). Curvilinear motion in the absence of external forces: Naive beliefs about the motion of objects. Science, 210(4474), 1139-1141.

McCloskey, M., Washburn, A., \& Felch, L. (1983). Intuitive physics: The straight-down belief and its origin. Journal of Experimental Psychology: Learning, Memory, and Cognition, 9(4), 636.

Papenmeier, F., Meyerhoff, H. S., Brockhoff, A., Jahn, G., \& Huff, M. (2017). Upside-down: Perceived space affects object-based attention. Journal of experimental psychology: human perception and performance, 43(7), 1269.

Pylyshyn, Z. W., \& Storm, R. W. (1988). Tracking multiple independent targets: Evidence for a parallel tracking mechanism. Spatial Vision, 3(3), 179-197.

Sanborn, A. N., Mansinghka, V. K., \& Griffiths, T. L. (2013). Reconciling intuitive physics and Newtonian mechanics for colliding objects. Psychological Review, 120(2), 411.

Scholl, B. J., \& Leslie, A. M. (1999). Explaining the infant's object concept: Beyond the perception/cognition dichotomy. In E. Lepore \& Z. Pylyshyn (Eds.), What is Cognitive Science? Oxford: Blackwell, 26-73.

Scimeca, J. M., \& Franconeri, S. L. (2015). Selecting and tracking multiple objects. Wiley Interdisciplinary Reviews: Cognitive Science, 6(2), 109-118.

Shim, W. M., Alvarez, G. A., \& Jiang, Y. V. (2008). Spatial separation between targets constrains maintenance of attention on multiple objects. Psychonomic Bulletin \& Review, 15(2), 390-397.

Smith, K. A., Battaglia, P. W., \& Vul, E. (2018). Different physical intuitions exist between tasks, not domains. Computational Brain \& Behavior, 1-18.

St. Clair, R. S., Huff, M., \& Seiffert, A. E. (2010). Conflicting motion information impairs multiple object tracking. Journal of Vision, 10(4), 18-18.

Stroop, J. R. (1935). Studies of interference in serial verbal reactions. Journal of Experimental Psychology, 18(6), 643.

Todd, J. T., \& Warren Jr, W. H. (1982). Visual perception of relative mass in dynamic events. Perception, 11(3), 325-335.

Tombu, M., \& Seiffert, A. E. (2008). Attentional costs in multiple-object tracking. Cognition, 108(1), 1-25. 
Vul, E., Frank, M. C., Alvarez, G., Tenenbaum, J. (2009). Explaining human multiple object tracking as resource-constrained approximate inference in a dynamic probabilistic model. Advances in Neural Information Processing Systems, 22.

Yantis, S. (1992). Multielement visual tracking: Attention and perceptual organization. Cognitive psychology, 24(3), 295-340.

Zhong, S. H., Ma, Z., Wilson, C., Liu, Y., \& Flombaum, J. I. (2014). Why do people appear not to extrapolate trajectories during multiple object tracking? A computational investigation. Journal of Vision, 14(12), 12-12. 
PHYSICS IN TRACKING

\section{Appendix}

\section{Part 1: Replication of Experiment 2}

To ensure the pattern found in Experiment 2 was reliable, we replicated the experiment with a new group of 40 participants. This group came from the same subject pool described in the main text. One participant was not able to finish the experiment within an hour, and her data were discarded, resulting in 39 participants in the analysis ( 37 female). The mean age of the participants was 19.8 years. Methods and the analysis scheme were adopted from Experiment 2.

\section{Results}

Overall performance was higher in the replication, compared to that in Experiment 2. Importantly, the general pattern of the results was replicated. Participants performed better in the realistic physics condition, compared to the constant speed condition, $\mathrm{t}(38)=5.73, p_{\mathrm{adj}}<0.001$. Performance in the constant speed and "Varied Speed, Incorrect Angle" conditions was indistinguishable, $\mathrm{t}(38)=0.17$, $p_{\text {adj }}=1$, Bayes Factor $=5.72$ in favor of the null hypothesis. Performance in the realistic physics and "Varied Speed, Correct Angle" conditions was also indistinguishable, $\mathrm{t}(38)=0.16, p_{\mathrm{adj}}=1$, Bayes Factor $=$ 5.72 in favor of the null hypothesis. The results are summarized in Figure S1. 
PHYSICS IN TRACKING

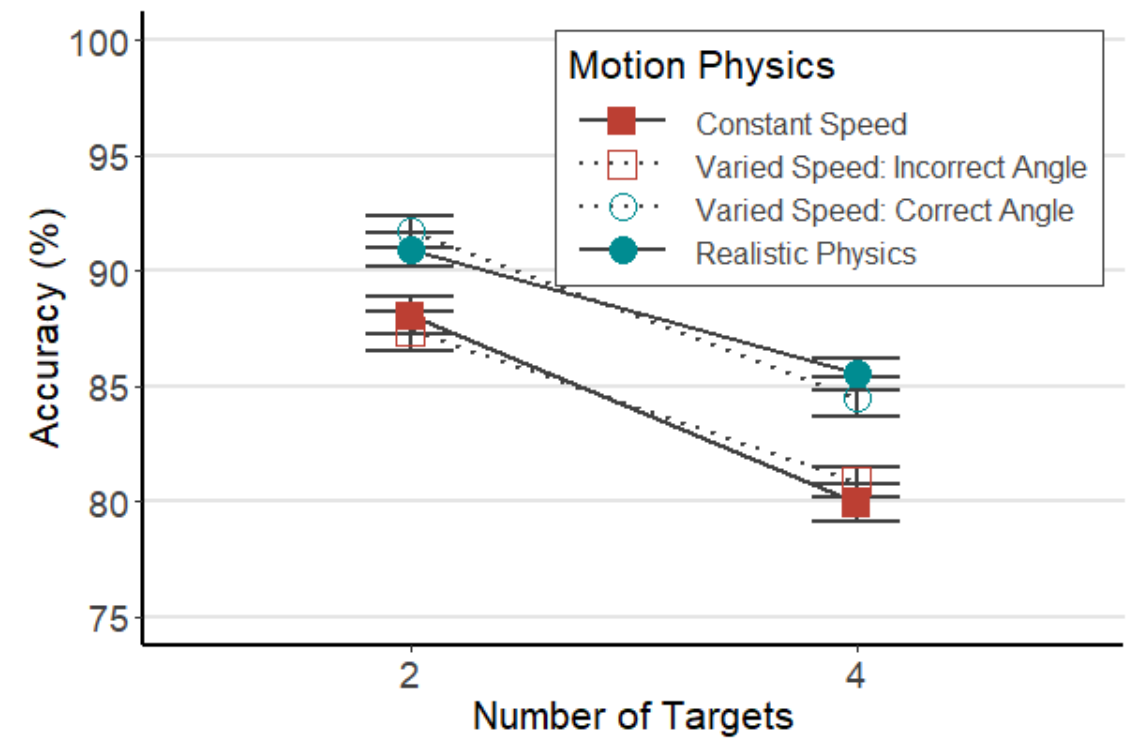

Figure S1. A replication attempt of Experiment 2. The pattern of the results was highly similar to that of Experiment 2, except that the overall performance was higher in the replication. Refer to the main text for methods. 


\section{Part 2: Pseudo-code for Computation of Reflection Angles}

The following snippet of pseudo-code illustrates how two colliding objects reflect from each other in

\section{Experiment 1.}

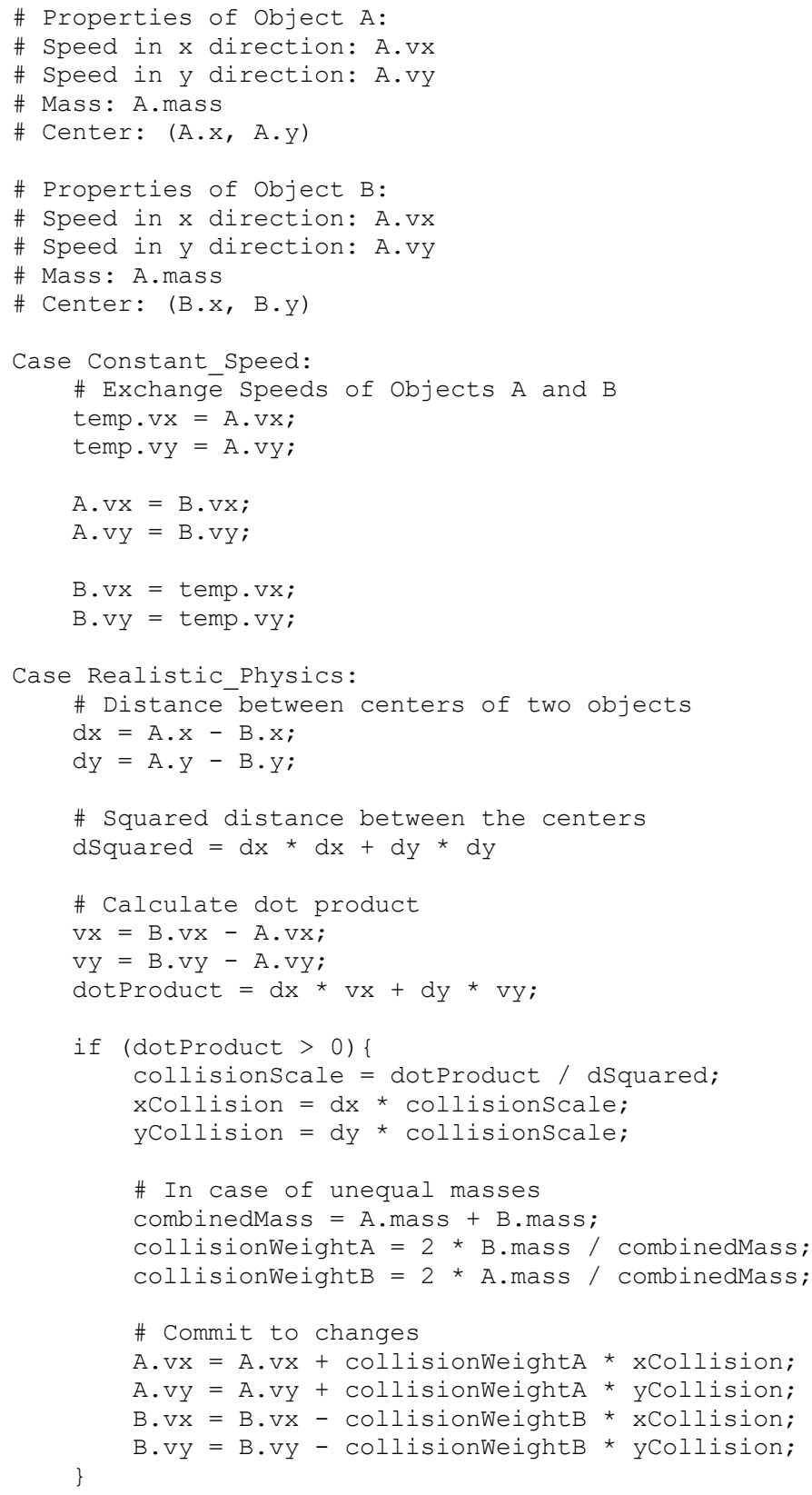

